\title{
PENTAQUARKS IN CHIRAL SOLITON MODELS ${ }^{1}$.
}

\author{
Vladimir B. Kopeliovich \\ Institute for Nuclear Research of Russian Academy of Sciences, \\ Moscow, 117312
}

\begin{abstract}
The spectra of pentaquarks, some of them being observed recently, are discussed within topological soliton model and compared with simplified quark picture. Results obtained within chiral soliton model depend to some extent on the quantization scheme: rigid rotator, soft rotator, or bound state model. The similarity of spectra of baryon resonances obtained within quark model and chiral soliton model is pointed out, although certain differences take place as well, which require careful interpretation. In particular, considerable variation of the strange antiquark mass in different $S U(3)$ multiplets of pentaquarks is required to fit their spectra obtained from chiral solitons. Certain difference of masses of "good" and "bad" diquarks is required as well, in qualitative agreement with previously made estimates. The partners of exotic states with different values of spin which belong to higher $S U(3)$ multiplets, have the energy considerably higher than states with lowest spin, and this could be a point where the difference from simple quark models is striking. The antiflavor excitation energies for multibaryons are estimated as well, and binding energies of $\Theta$-hypernuclei and anticharm (antibeauty) hypernuclei are presented for several baryon numbers. Some deficiencies in the argumentation against validity of the chiral soliton approach and/or $S U(3)$ quantization models, existing in the literature, are pointed out.
\end{abstract}

\section{Introduction}

Recent events around possible discovery of pentaquarks and negative results, obtained lately, make situation with the observation of these new type baryonic resonances quite dramatic. Indeed, one of striking events in elementary particle physics of the last few years was observation of baryon resonances with unusual properties (end of 2002 - 2005):

$\Theta^{+}(1540)$, strangeness $S=+1$, isospin $I=0$ (most likely), width $\Gamma_{\Theta}<10 \mathrm{MeV}$, seen by different collaborations in Japan, Russia, USA, FRG, CERN;

$\Phi / \Xi_{3 / 2}^{--}(1862)$, strangeness $S=-2, \quad I=3 / 2(?), \Gamma<18 \mathrm{MeV}$ observed by NA49 Collab. at $\mathrm{CERN}^{2}$;

$\Theta_{c}^{0}(3099)$, charm $C=-1, \Gamma<15 \mathrm{MeV}$ seen by H1 Collab., DESY. Spin-parity $J^{P}$ of these states is not measured yet.

These states are manifestly exotic because they cannot be made of 3 valence quarks only. There are different possibilities to have exotic baryon states:

a) positive strangeness $S>0$ (or negative charm $C<0$, or positive beauty), since $s$-quark has $S=-1$ and $c$-quark $C=+1$,

b) large (in modulus) negative strangeness $S<-3 B, B$ - baryon number; similar for charm or beauty,

c) large enough isospin $I>(3 B+S) / 2$, if $-3 B \leq S \leq 0$, or charge $Q>2 B+S$ or $Q<-B$ in view of Gell-Mann - Nishijima relation $Q=I_{3}+Y / 2$.

\footnotetext{
${ }^{1}$ Based partly on the talks presented at the International Seminar on High Energy Physics Quarks2004, Pushkinogorie, Russia, May 24-30, 2004; International Workshop on Quantum Field Theory and High Energy Physics QFTHEP-04, Saint-Petersburg, Russia, June 17-23, 2004, and Symposium of London Mathematical Society "Topological Solitons and their Applications", Durham, UK, August 2-12, 2004. Slightly reduced version of this paper is available as E-print hep-ph/0507028.

${ }^{2}$ In the latest issues of PDG the state $\Xi_{3 / 2}$ is called $\Phi$ [1], although the notation $\Xi_{3 / 2}$ seems to be more informative; here it will be denoted as $\Phi / \Xi_{3 / 2}$
} 
The pentaquarks $\Theta^{+}(1540)$ and $\Theta_{c}(3099)$, if it is confirmed, are just of the type a), the possibility $\mathbf{b})$ is difficult to be realized in practice, since large negative strangeness of produced baryon should be balanced by corresponding amount of positively strange kaons; high enough energy of colliding particles is necessary here. The state $\Phi / \Xi_{3 / 2}^{--}$is of the type $\mathbf{c}$ ).

The minimal quark contents of these states are:

$\Theta^{+}=(d d u u \bar{s}) ; \quad \Xi^{--}=(s s d d \bar{u}) ; \Theta_{c}^{0}=(d d u u \bar{c})$, and by this reason they are called pentaquarks.

The history and chronology of pentaquarks predictions and discovery has been discussed already in many papers, here I recall it briefly for completeness. Readers familiar with this subject can go immediately to sections 3,4 .

$\Theta^{+}, S=+1$ baryon was observed first at SPring-8 installation (RCNP, Japan)[2] in reaction $\gamma^{12} \mathrm{C} \rightarrow K^{+} n+\ldots$. The reported mass is $1540 \pm 10 \mathrm{MeV}$ and width $\Gamma<25 \mathrm{MeV}$, confidence level (CL) $4.6 \sigma$. Soon after this and independently DIANA collaboration at ITEP, Moscow [3] reported on observation of $\Theta^{+}$state in interactions of $K^{+}$in $X e$ bubble chamber. The mass of the bump in $K^{0} p$ invariant mass distribution is $1539 \pm 2 \mathrm{MeV}, \Gamma<$ $9 \mathrm{MeV}$, confidence level a bit lower, about $4.4 \sigma$.

Confirmation of this result came also from several other experiments $[4,5,6,7,8,9$, $10,11,12]$ mostly in reactions of photo-(electro)-production. The reported mass of $\Theta^{+}$ is smaller by several $\mathrm{MeV}$, or even by $\sim(10-20) \mathrm{MeV}$ in some of experiments $[6,8,9$, 10, 11], than first reported value $1540 \mathrm{MeV}$ [2]. The CLAS Collaboration [13] provided recently evidence for two states in $\Theta$-region of the $K^{+} n$ invariant mass distribution at $1523 \pm 5 \mathrm{MeV}$ and $1573 \pm 5 \mathrm{Mev}$. The nonobservation of $\Theta^{+}$in old kaon-nucleon scattering data provided restriction on the width of this state. Phase shift analysis of $K N$-scattering in the energy interval $1520-1560 \mathrm{MeV}$ gave a restriction $\Gamma<1 \mathrm{MeV}$ [14]. Later analysis of data [3], obtained in $X e$ bubble chamber, allowed to get the estimate for the width of $\Theta^{+}: \Gamma_{\Theta} \simeq 0.9 \pm 0.3 \mathrm{MeV}$, and from total cross section data $\Gamma_{\Theta}$ in the interval $1-4 \mathrm{MeV}$ [15].

Several experiments, mostly at higher energies, did not confirm existence of $\Theta$, pessimistic point of view was formulated, e.g., in [16]. More complete list of references to negative results, many of them being not published yet, and their critical discussion can be found in $[17,18,19]$. Most serious seems to be recent negative result on $\Theta^{+}$photoproduction on protons, obtained at spectrometer CLAS (JLAB) with high statistics, where no resonance has been observed in $K^{+} n$ final state in the mass interval $1520-1600 \mathrm{MeV}$ scanned with $5 \mathrm{MeV}$ steps [20].

The doubly strange cascade hyperon $\Phi / \Xi_{3 / 2}, S=-2$, probably with isospin $I=3 / 2$, is observed in one experiment at CERN, only, in proton-proton collisions at $17 \mathrm{GeV}[21]$. The mass of resonance in $\Xi^{-} \pi^{-}$and $\bar{\Xi}^{+} \pi^{+}$systems is $1862 \pm 2 \mathrm{MeV}$ and mass of resonance in $\Xi^{-} \pi^{+}, \bar{\Xi}^{+} \pi^{-}$systems is $1864 \pm 5 \mathrm{MeV}$, width $\Gamma<18 \mathrm{MeV}$, and $C L=4.0 \sigma$. The fact makes this result more reliable, that resonance $\Phi / \Xi^{--}$was observed in antibaryon channel as well. However, this resonance is not confirmed by HERA-B, ZEUS, CDF, WA89, COMPASS collaborations (see e.g. [16, 22]), although there is no direct contradiction with NA49 experiment because other reactions have been used and mostly at higher energies, so, upper bounds on the production cross sections of $\Phi / \Xi_{3 / 2}$ have been obtained in this way, see $[22,23]$ for compilation of these results.

The anticharmed pentaquark $\Theta_{c}^{0}, C=-1$ was observed at H1, HERA, Germany, in ep collisions [24], in both baryon number $B= \pm 1$ channels. The mass of resonance in $D^{*-} p, D^{*+} \bar{p}$ systems is $3099 \pm 3 \pm 5 \mathrm{MeV}, \Gamma<15 \mathrm{MeV}, C L=6.2 \sigma$. ZEUS collaboration at HERA [25] did not confirm the existence of $\Theta_{c}^{0}$ with this value of mass, and this seems to be serious contradiction, see again [16].

There is also some evidence for existence of other baryon states, probably exotic, e.g. nonstrange state decaying into nucleon and two pions [26], and resonance in $\Lambda K_{S}^{0}$ system 
with mass $1734 \pm 6 \mathrm{MeV}, \Gamma<6 \mathrm{MeV}$ at $C L=(3-6) \sigma$ which is $N^{* 0}$ or $\Xi_{1 / 2}^{*}$, observed by STAR collaboration at RHIC [27] in reaction $A u+A u$ at $\sqrt{s_{N N}} \sim 200 \mathrm{GeV}$. Several resonances in $\Lambda K_{S}^{0}$ system have been observed recently at JINR [28], the lowest one has the mass $1750 \pm 18 \mathrm{MeV}$, not in contradiction with [27].

Evidently, high statistics experiments on pentaquarks production are drastically needed, as well as checking the relatively old scattering data analyzed in $[14,15]$. If high statistics experiments do not confirm existence of $\Theta^{+}$, it would be interesting then to understand why more than 10 different experiments, although each of them with not high statistics, using different installations and incident particles, provided similar positive results. From theoretical point of view the interest to such exotic baryon states will not dissappear in any case, because they represent the next in complexity step after baryons made of 3 valence quarks and should most probably appear at higher masses and with greater widths.

Information about status of higher statistics experiments performed or to be performed at JLAB (CLAS Collab.) can be found in [29, 19]. Several reviews of existing experimental situation appeared lately, e.g. [22, 23, 18, 19, 30], and I will not go into further details here. In any case, the difficulties in observation of such exotic states mean that the role of these states in hadron dynamics of moderate and especially high energies is not big and cannot be even compared with, e.g., the role of $\Delta(1232)$ resonance in medium energy pion-nucleon interactions.

Next sections necessarily contain certain overlap with previous discussions, I hope to add some new accent of criticism to the discussion of this interesting topic. Sections 3,4 contain short description of the topological soliton models, in section 5 the results for baryon spectra are presented. In Section 6 the large $N_{c}$ arguments that the width of $\Theta^{+}$is expected to be greater than width of $\Delta$ resonance are criticized and ambiguity of large $N_{c}$ consideration is stressed, in Section 7 a correspondence with the quark model description is established and some difference is fixed and discussed as well. In section 8 the masses of partners of lowest pentaquark states with different spin or isospin are estimated. In Section 9 the multibaryons with additional quark-antiquark pairs are discussed within chiral soliton approach, which can appear as $\Theta$ hypernuclei, or hypernuclei with anticharm (-beauty), and it is argued that existence of such states is a natural property of this approach. Section 10 contains conclusions and some prospects.

\section{Early predictions}

From theoretical point of view the existence of such exotic states by itself was not unexpected. Such states have been discussed first by R.L.Jaffe within MIT quark-bag model [31]. The mass of these states was estimated to be considerably higher than that reported now: $M_{\Theta} \simeq 1700 \mathrm{MeV}, J^{P}=1 / 2^{-}$. These studies were continued by other authors [32]: $M_{\Theta} \simeq 1900 \mathrm{MeV}, J^{P}=1 / 2^{-}$, and similar in [33]. From analysis of existed that time data on $K N$ interactions the estimate was obtained in [34] $M_{\Theta} \simeq 1705 \mathrm{MeV}(I=0), 1780 \mathrm{MeV}(I=1)$ with very large width. If the data on narrow low lying pentaquarks are not confirmed, then these earlier predictions have more chances to be correct.

In the context of chiral soliton model the $\{\overline{10}\}$ and $\{27\}$-plets of exotic baryons were mentioned first in [35], without any mass estimates, however. Rough estimate within the "toy" model, $M_{\overline{10}}-M_{8} \simeq 600 \mathrm{MeV}$, was made a year later in [36]. A resonance-like behaviour of $K N$ scattering phase shift in $\Theta$ channel was obtained in [37] in a version of Skyrme model (in the limit $\left.M_{K}=M_{\pi}\right)^{3}$.

\footnotetext{
${ }^{3}$ Recently this result has been criticized in [68].
} 
Numerical estimate $M_{\Theta} \simeq 1530 \mathrm{MeV}$ was obtained first by M.Praszalowicz [38]. The mass splittings within octet and decuplet of baryons have not been described satisfactorily with parameters of the model accepted that time, but in the "flexible" approach proposed in [38] 8 masses of octet and decuplet of baryons were fitted with Skyrme model motivated mass formula, depending on 4 parameters, defined from this fit. Central value of the mass of antidecuplet was found equal to $1706 \mathrm{MeV}$.

Extension of quantization condition [39] to "exotic" case was made in [40] where masses of exotic baryonic systems ( $B$ arbitrary, $N_{c}=3$ ) were estimated as function of the number of additional quark-antiquark pairs m: $\Delta E \sim m / \Theta_{K}, m^{2} / \Theta_{K}$. It was neither mass splittings estimates inside of multiplets, nor calculations of masses of particular exotic baryons in [40], although it was shown that baryonic states with additional quarkantiquark pairs appear quite naturally within chiral soliton approach as $S U(3)$ rotational excitations. Recently more general consideration of such states has been performed in [41] for arbitrary numbers of colors and flavors.

First calculation with configuration mixing due to flavor symmetry breaking $\left(m_{K} \neq\right.$ $m_{\pi}$ ) was made by H.Walliser in [42] where mass splittings within the octet and decuplet of baryons were well described, and estimate obtained $M_{\Theta} \simeq 1660 \mathrm{MeV}$. "Strange" or kaonic inertia $\Theta_{K}$ which governs the mass splitting between exotic and nonexotic baryon multiplets was underestimated in this work, as it is clear now (see below).

The estimate $M_{\Theta} \simeq 1530 \mathrm{MeV}$, coinciding with [38], and first estimate of the width, $\Gamma_{\Theta}<30 \mathrm{MeV}$ were made later by D.Diakonov, V.Petrov and M.Polyakov [43] in a variant of quark-soliton model. It was "a luck", as stated much later by same authors: mass splitting inside of $\overline{10}$ was obtained equal to $540 \mathrm{MeV}$, greater than for decuplet of baryons, and it was supposed that resonance $N^{*}(1710) \in\{\overline{10}\}$, i.e. it is the nonstrange component of antidecuplet. The above mass value of $\Theta^{+}$was a result of subtraction, $1530=1710-$ 540/3. However, the paper [43], being in right direction, stimulated successful (as we hope still!) searches for $\Theta^{+}$in RCNP (Japan) [2] and ITEP (Russia) [3].

Skyrme-type model with vibrational modes included was studied in details first by H.Weigel [44] with a result $M_{\Theta} \simeq 1570 \mathrm{MeV}, \Gamma_{\Theta} \sim 70 \mathrm{MeV}$. An inconsistency in width estimate of [43] was noted here.

\section{$\underline{\text { Developements after } \Theta^{+} \text {discovery. }}$}

After discovery of pentaquarks there appeared big amount of papers on this subject which develop theoretical ideas in different directions: within chiral soliton models [45, $46,47,48,49,50,51,52,53]$ and many other; phenomenological correlated quark models $[54,55,56,57,58]$ and other, critical discussion by F.Close can be found in [59]; QCD sum rules $[60,61]$; by means of lattice calculations $[62,63]$, etc. It is not possible to describe all of them within restricted framework of present paper (reviews of that topic from different sides can be found, e.g. in [64, 65]). Quite sound criticism concerning rigid rotator quantization within chiral soliton models was developed in [66, 67, 68], but it should be kept in mind that the drawbacks of soliton approach should be compared with uncertainties and drawbacks of other models ${ }^{4}$. There is no regular way of solving relativistic many-body problem to find bound states or resonances in 3-,5-, etc. quark system, and the chiral soliton approach, in spite of its drawbacks, provides a way to circumwent some of difficulties. The correlated quark models, diquark-triquark model [54], or diquark-diquark-antiquark model [55], being interesting and predictive, contain certain, and very substantial, phenomenological assumptions.

\footnotetext{
${ }^{4}$ It is stated in conclusions of second of papers [66]: "this paper does not show that rigid-rotor quantization is necessarily invalid but rather that it is not justified due to large $N_{c}$ QCD. It remains possible that it is justified due to some other reason". We agree with this rather optimistic conclusion.
} 


\section{Topological soliton model}

In spite of some uncertainties and discrepances between different authors, the chiral soliton approach provided predictions for the masses of exotic states near the value observed later, considerably more near than quark or quark-bag models made up to that time. Here I will be restricted with this model, mainly. Situation is somewhat paradoxical: it is easier to estimate masses of exotic states within chiral soliton models, whereas interpretation is more convenient in terms of simplified quark model.

The topological soliton model is very elegant and attractive (to authors opinion) since it allows to consider the families ( $S U(3)$-multiplets) of baryons, nonexotic and exotic, in unique way. At the same time, as mentioned in literature, see e.g. [55], the apparent drawback is that this approach does not predict anything about exotics in meson sector. In these models the baryons and baryonic systems appear as classical configurations of chiral ("pionic" in simplest $S U(2)$ version) fields which are characterized by the topological or winding number identified with the baryon number of the system [69]. This baryon number is the 4-th component of the Noether current generated by the Wess-Zumino term in the action written in a compact form by Witten [70], I shall not reproduce it here. In other words, the B-number is degree of the map $R^{3} \rightarrow S U(2)$, or $R^{3} \rightarrow S^{3}$, since $S U(2)$ is homeomorphic to 3 -dimensional sphere $S^{3}$ :

$$
B=\frac{-1}{2 \pi^{2}} \int s_{f}^{2} s_{\alpha} I\left[\frac{(f, \alpha, \beta)}{(x, y, z)}\right] d^{3} r
$$

where functions $f, \alpha, \beta$, describing $S U(2)$ skyrmion, define the direction of unit vector $\vec{n}$ on 3-dimensional sphere $S^{3}$ and $I[(f, \alpha, \beta) /(x, y, z)]$ is Jacobian of corresponding transformation. More details can be found, e.g. in [70, 45, 65], see also (2) below. It is important that the number of dimensions of the ordinary space, equal to 3 , coincides with the number of degrees of freedom (or generators) of the $S U(2)$ group, and this makes possible the mapping ordinary space onto isospace. This can be an explanation why the isospin symmetry takes place in hadronic world.

The effective chiral lagrangian describing low-energy phenomena can be obtained from underlying QCD by means of special procedure of bosonization $[71,72,73]$, it contains infinite number of terms - powers of chiral derivatives, and, as it is believed, is equivalent to underlying QCD. Many known features of low energy meson-meson and meson-baryon interactions found explanation within this effective theory. In soliton models the truncated lagrangian is used as starting point - few first terms of this expansion are taken into account to insure solitons stabilization by 4 -th, sometimes 6 -th order term in chiral derivatives [74], and to make evaluations technically possible. Further progress in this direction is discussed in $[75,76]$.

The Lagrangian density of the model in its minimal form is

$$
L=-\frac{F_{\pi}^{2}}{16} \operatorname{Tr}\left(l_{\mu} l_{\mu}\right)+\frac{1}{32 e^{2}} \operatorname{Tr}\left[l_{\mu} l_{\nu}\right]^{2}+\frac{F_{\pi}^{2} m_{\pi}^{2}}{16} \operatorname{Tr}\left(U+U^{\dagger}-2\right),
$$

where $m_{\pi}, F_{\pi}$ are pion mass and decay constant taken from experiment, $e$ is the Skyrme parameter defining the weight of the 4-th order term, stabilizing the soliton, $e \sim 4$, according to most of latest estimates, which allows to describe the mass splittings of octet and decuplet of baryons; $l_{\mu}=\partial_{\mu} U U^{\dagger}, U$ is unitary matrix incorporating the chiral fields, in $S U(2)$ case $U=c_{f}+i \vec{n} \vec{\tau}$ and 3 components of the unit vector $\vec{n}$ are $s_{\alpha} c_{\beta}, s_{\alpha} s_{\beta}, c_{\alpha}$, see Eq. (1) above. For each value of baryon number, one should find the classical field configuration of minimal energy (mass) - this is done often by means of variational minimization numerical codes. For $B=1$ configuration of minimal energy is of so called "hedgehog" type, where chiral field at each space point can be directed along radius vector drawn 
from center of soliton $(\vec{n}=\vec{r} / r$, for $B=2$ it has torus-like form, for $B=3$ it has topology of tetrahedron, etc. The relation takes place for configurations of minimal energy between second order term, fourth order term and the mass term (M.t.) contributions to classical mass of solitons (known as Derrick theorem)

$$
M^{(2)}+3 M . t .=M^{(4)} .
$$

The next step is the quantization of these configurations to get spectrum of states with definite quantum numbers, isospin $I$, strangeness $S$ or hyperchrge $Y$. At this point the flavor symmetry breaking $(F S B)$ terms in the Lagrangian are important which define the mass splittings within $S U(3)$ multiplets of baryons or baryonic systems. Usually they are taken in the form

$L_{F S B}=\frac{F_{K}^{2} m_{K}^{2}-F_{\pi}^{2} m_{\pi}^{2}}{24} \operatorname{Tr}\left(1-\sqrt{3} \lambda_{8}\right)\left(U+U^{\dagger}-2\right)-\frac{F_{K}^{2}-F_{\pi}^{2}}{48} \operatorname{Tr}\left(1-\sqrt{3} \lambda_{8}\right)\left(U l_{\mu} l_{\mu}+l_{\mu} l_{\mu} U^{\dagger}\right)$

In the collective coordinates quantization procedure $[77,39]$ one introduces the angular velocities of rotation of skyrmion in the $S U(3)$ configuration space, $\omega_{k}, k=1, \ldots 8$ : $A^{\dagger}(t) \dot{A}(t)=-i \omega_{k} \lambda_{k} / 2, \lambda_{k}$ being Gell-Mann matrices, the collective coordinates matrix $A(t)$ is written usually in the form $A=A_{S U 2} \exp \left(i \nu \lambda_{4}\right) A_{S U 2}^{\prime} \exp \left(i \rho \lambda_{8} / \sqrt{3}\right)$. The WessZumino term contribution into lagrangian can be calculated explicitly for this ansatz, $L_{W Z}=-\omega_{8} N_{c} B / 2 \sqrt{3}$, and so called "right" hypercharge, or hypercharge in the bodyfixed system equals $Y_{R}=-2 \partial L / \partial \omega_{8} / \sqrt{3}=N_{c} B / 3$. For any $S U(3)$ multiplet $(p, q)$ the maximal hypercharge $Y_{\max }=(p+2 q) / 3$, and obviously, inequality should be fulfilled $p+2 q \geq N_{c} B$, or

$$
p+2 q=3(B+m)
$$

for $N_{c}=3$, with $m$ positive integer. States with $m=0$ can be called, naturally, minimal multiplets. For $B=1$ they are well known octet $(1,1)$ and decuplet $(3,0)$ [39].

States with $m=1$ should contain at least one $q \bar{q}$ pair, since they contain the $S=$ $+1, Y=2$ hyperon. They are pentaquarks antidecuplet $(p, q)=(0,3), 27$-plet $(2,2)$, 35-plet $(4,1)$. The pentaquark multiplets are presented in Figure. The minimal value of hypercharge is $Y_{\min }=-(2 p+q) / 3$, the maximal isospin $I_{\max }=(p+q) / 2$ at $Y=(p-q) / 3$. Such multiplets as $\{27\},\{35\}$ for $m=1$ and multiplets for $m=2$ in their internal points contain 2 or more states (shown by double or triple circles in Figure). The 28-plet $(6,0)$ should contain at least two quark-antiquark pairs, as it follows from analyses of its strangeness and isospin content [65], so, it is septuquark (or heptaquark), although it has $m=1$, and it is not shown here by this reason.

\section{The mass formula for the rigid rotator}

The lagrangian describing baryons or baryonic system is quadratic form in angular velocities defined above, with momenta of inertia, isotopical (pionic) $\Theta_{\pi}$ and flavor, or kaonic $\Theta_{K}$ as coefficients [39]:

$$
L_{r o t}=\frac{1}{2} \Theta_{\pi}\left(\omega_{1}^{2}+\omega_{2}^{2}+\omega_{3}^{2}\right)+\frac{1}{2} \Theta_{K}\left(\omega_{4}^{2}+\ldots+\omega_{7}^{2}\right)-\frac{N_{c} B}{2 \sqrt{3}} \omega_{8} .
$$

The expressions for these moments of inertia as functions of skyrmion profile are well known [77, 39] and presented in many papers, see e.g. [65]. The quantization condition (5) discussed above follows from the presence of linear in angular velocity $\omega_{8}$ term in (3) originated from the Wess-Zumino-Witten term in the action of the model [70, 39]. 
The hamiltonian of the model can be obtained from (3) by means of canonical quantization procedure [39]:

$$
H=M_{c l}+\frac{1}{2 \Theta_{\pi}} \vec{R}^{2}+\frac{1}{2 \Theta_{K}}\left[C_{2}\left(S U_{3}\right)-\vec{R}^{2}-\frac{N_{c}^{2} B^{2}}{12}\right],
$$

where the second order Casimir operator for the $S U(3)$ group, $C_{2}\left(S U_{3}\right)=\sum_{a=1}^{8} R_{a}^{2}$, with eigenvalues for the $(p, q)$ multiplets $C_{2}\left(S U_{3}\right)_{p, q}=\left(p^{2}+p q+q^{2}\right) / 3+p+q$, for the $S U(2)$ group, $C_{2}(S U 2)=\vec{R}^{2}=R_{1}^{2}+R_{2}^{2}+R_{3}^{2}=J(J+1)=I_{R}\left(I_{R}+1\right)$.

The operators $R_{\alpha}=\partial L / \partial \omega_{\alpha}$ satisfy definite commutation relations which are generalization of the angular momentum commutation relations to the $S U(3)$ case [39]. Evidently, the linear in $\omega$ terms in lagrangian (6) are cancelled in hamiltonian (7). The equality of angular momentum (spin) $J$ and the so called right or body fixed isospin $I_{R}$ used in (7) takes place only for configurations of the "hedgehog" type when usual space and isospace rotations are equivalent. This equality is absent for configurations which provide the minimum of classical energy for greater baryon numbers, $B \geq 2$ [78].

\begin{tabular}{|l|l|l|l|l|l|l|}
\hline$(p, q)$ & $N(p, q)$ & $\mathrm{m}$ & $C_{2}\left(S U_{3}\right)$ & $J=I_{R}$ & $K\left(J_{\max }\right)$ & $K\left(J_{\max }-1\right)$ \\
\hline$(1,1)$ & $\{8\}$ & 0 & 3 & $1 / 2$ & $3 / 2$ & \\
$(3,0)$ & $\{10\}$ & 0 & 6 & $3 / 2$ & $3 / 2$ & \\
\hline$(0,3)$ & $\{\overline{10}\}$ & 1 & 6 & $1 / 2$ & $3 / 2+3$ & \\
$(2,2)$ & $\{27\}$ & 1 & 8 & $3 / 2 ; 1 / 2$ & $3 / 2+2$ & $3 / 2+5$ \\
$(4,1)$ & $\{35\}$ & 1 & 12 & $5 / 2 ; 3 / 2$ & $3 / 2+1$ & $3 / 2+6$ \\
$(6,0)$ & $\{28\}$ & 1 & 18 & $5 / 2$ & $3 / 2+7$ & \\
\hline$(1,4)$ & $\{\overline{35}\}$ & 2 & 12 & $3 / 2 ; 1 / 2$ & $3 / 2+6$ & $3 / 2+9$ \\
$(3,3)$ & $\{64\}$ & 2 & 15 & $5 / 2 ; 3 / 2 ; 1 / 2$ & $3 / 2+4$ & $3 / 2+9$ \\
$(5,2)$ & $\{81\}$ & 2 & 20 & $7 / 2 ; 5 / 2 ; 3 / 2$ & $3 / 2+2$ & $3 / 2+9$ \\
$(7,1)$ & $\{80\}$ & 2 & 27 & $7 / 2 ; 5 / 2$ & $3 / 2+9$ & $3 / 2+16$ \\
$(9,0)$ & $\{55\}$ & 2 & 36 & $7 / 2$ & $3 / 2+18$ & \\
\hline
\end{tabular}

Table 1.The values of $N(p, q)$, Casimir operator $C_{2}\left(S U_{3}\right)$, spin $J=I_{R}$, coefficient $K$ for first two values of $J$ for minimal $(m=0)$ and nonminimal $(m=1,2)$ multiplets of baryons.

For minimal multiplets $(m=0)$ the right isospin $I_{R}=p / 2$, and it is easy to check that coefficient of $1 / 2 \Theta_{K}$ in (7) equals to

$$
K=C_{2}\left(S U_{3}\right)-\vec{R}^{2}-N_{C}^{2} B^{2} / 12=N_{C} B / 2,
$$

for arbitrary $N_{C}{ }^{5}$. So, $K$ is the same for all multiplets with $m=0$ [40], see Table 1 the property known long ago for the $B=1$ case [39]. For nonminimal multiplets there are additional contributions to the energy proportional to $m / \Theta_{K}$ and $m^{2} / \Theta_{K}$, according to $(7)$. The following expression was obtained for the energy surplus due to addition of $m$ quark-antiquark pairs (formula (6) of [40]):

$$
\delta E_{\text {rot }}=m\left[(3 B / 2+1+m-N) /\left(2 \Theta_{K}\right)+(2 N+1-m) /\left(2 \Theta_{\pi}\right)\right]
$$

\footnotetext{
${ }^{5}$ For the number of colors $N_{C}$ different from 3 the minimal multiplets for baryons differ from octet and decuplet. They have $[p, q]=\left[1,\left(N_{c}-1\right) / 2\right],\left[3,\left(N_{c}-3\right) / 2\right], \ldots,\left[N_{c}, 0\right]$. There are totally $\left(N_{c}+1\right) / 2$ multiplets of baryons with the interval $\Delta Y$ for each of them increasing from $\left(N_{c}+1\right) / 2$ to $N_{c}$, see Appendix. It is an artifact of large $N_{c}$ approximation that besides the states existing in real world with $N_{c}=3$, the spurious states appear, and the number of these states is infinite as $N_{c} \rightarrow \infty$.
} 
with "right isospin" $N=(p+m) / 2$. It means that in the framework of chiral soliton approach the "weight" of quark- antiquark pair is defined by parameter $1 / \Theta_{K}$, and this property of such models deserves better understanding.

Table 1 was presented previously in [65], here we need it also to estimate the mass difference of partners of lowest baryon states with same quantum numbers except spin. It follows from Table 1 that for each nonzero $m$ the coefficient $K\left(J_{\max }\right)$ decreases with increasing $N(p, q)$, e.g. $K_{5 / 2}(35)<K_{3 / 2}(27)<K_{1 / 2}(\overline{10})$. The following differences of the rotation energy can be obtained easily:

$$
M_{10}-M_{8}=\frac{3}{2 \Theta_{\pi}}, \quad M_{\overline{10}}-M_{8}=\frac{3}{2 \Theta_{K}},
$$

obtained in [39, 43],

$$
\begin{gathered}
M_{27, J=3 / 2}-M_{10}=\frac{1}{\Theta_{K}}, \quad M_{27, J=3 / 2}-M_{\overline{10}}=\frac{3}{2 \Theta_{\pi}}-\frac{1}{2 \Theta_{K}}, \\
M_{27, J=3 / 2}-M_{8}=\frac{1}{\Theta_{K}}+\frac{3}{2 \Theta_{\pi}}
\end{gathered}
$$

which follows also from (9) at $B=1, m=1$,

$$
M_{35, J=5 / 2}-M_{27, J=3 / 2}=\frac{5}{2 \Theta_{\pi}}-\frac{1}{2 \Theta_{K}}
$$

According to these relations, the mass difference between decuplet and 27-plet is 1.5 times smaller than between octet and antidecuplet, by this reason the mixing between corresponding components of decuplet and 27-plet is large and not negligible, although it was neglected in [43, 79], e.g. If the relation took place $\Theta_{K} \ll \Theta_{\pi}$, then $\{27\}$-plet would be lighter than antidecuplet, and $\{35\}$-plet would be lighter than $\{27\}$-plet. In realistic case $\Theta_{K}$ is approximately twice smaller than $\Theta_{\pi}$ (see Table 2 , next section), and therefore the components of antidecuplet are lighter than components of $\{27\}$ with same values of strangeness. Beginning with some values of $N(p, q)$ coefficient $K$ increases strongly, as can be seen from Table 1, and this corresponds to the increase of the number of quarkantiquark pairs by another unity. The states with $J<J_{\max }$ have the energy considerably greater than that of $J_{\max }$ states, by this reason they could contain also greater amount of $q \bar{q}$-pairs.

The states with $m=2$ (second floor of this building) have considerably higher energy than states with $m=1$, and this difference defines the scale of mass splitting between partners with higher values of spin. It can be noted from Table 1 that the 35-plet with $J=3 / 2$ has exactly the same rotation energy as $\overline{35}$-plet, $(m=2)$ with $J=3 / 2$ : $K=3 / 2+6$ in both cases (this degeneracy was noted first by H.Walliser). It is a clear hint that states with same values of $(p, q)$ but different spin should have different quark contents.

The formula (7) is obtained in the rigid rotator approximation which is valid if the profile function of the skyrmion and therefore its dimensions and other properties are not changed when it is rotated in the configuration space (see next section and, e.g. discussion in $[65])$.

\section{$5 \quad$ Spectrum of baryonic states}

Expressions (7-13) and numbers given in Table $\mathbf{1}$ are sufficient to calculate the spectrum of baryons without mass splitting inside of $S U(3)$ - multiplets, as it was made e.g. in $[36,40]$. 


\subsection{The rigid rotator model}

The mass splitting due to the presence of flavor symmetry breaking terms in the lagrangian (4) plays a very substantial role in the spectrum of baryon states. The corresponding contribution into hamiltonian can be written in simple form [84, 42, 45]:

$$
H_{S B}=\frac{1-D_{88}^{(8)}}{2} \Gamma_{S B}=\frac{3}{4} s_{\nu}^{2} \Gamma_{S B}
$$

where the $S U(3)$ rotation function $D_{88}^{8}(\nu)=1-3 s_{\nu}^{2} / 2$,

$$
\begin{gathered}
\Gamma_{S B}=\frac{2}{3}\left[\left(\frac{F_{K}^{2}}{F_{\pi}^{2}} m_{K}^{2}-m_{\pi}^{2}\right) \Sigma+\left(F_{K}^{2}-F_{\pi}^{2}\right) \tilde{\Sigma}\right] \\
\Sigma=\frac{F_{\pi}^{2}}{2} \int\left(1-c_{f}\right) d^{3} \vec{r}, \quad \tilde{\Sigma}=\frac{1}{4} \int c_{f}\left(f^{\prime 2}+\frac{2 s_{f}^{2}}{r^{2}}\right) d^{3} r,
\end{gathered}
$$

kaon and pion masses $m_{K}, m_{\pi}$ as well as $F_{K}, F_{\pi}$ are taken from experiment. At large number of colors $\Gamma_{S B} \sim N_{c}$, both the soliton mass and the total mass splitting of $S U(3)$ multiplets also are $\sim N_{c}$. Individual mass splittings within multiplets are of the order of $N_{c}^{0} \sim 1$, since the change of hypercharge within multiplets $\Delta Y \sim N_{c}$, see footnote in previous section. The quantity $S C=<s_{\nu}^{2}>/ 2=<1-D_{88}^{(8)}>/ 3$ averaged over the baryon $S U(3)$ wave function defines its strangeness content, which allows to establish a bridge between chiral soliton approach and quark models ${ }^{6}$. Without configuration mixing, i.e. when flavor symmetry breaking terms in the lagrangian are considered as small perturbation, $\left\langle s_{\nu}^{2}>_{0}\right.$ can be expressed simply in terms of the $S U(3)$ ClebshGordan coefficients. The values of $\left\langle s_{\nu}^{2}\right\rangle_{0}$ for the octet, decuplet, antidecuplet and some components of higher multiplets are presented in Tables 2, 5. In this approximation the components of $\{10\}$ and $\{\overline{10}\}$ are placed equidistantly, and splittings of decuplet and antidecuplet are equal.

The spectrum of states with configuration mixing and diagonalization of the hamiltonian in the next orders of perturbation theory in $H_{S B}$ is given in Table 2 (the code for calculation was kindly presented by H.Walliser). The calculation results in the Skyrme model with only one adjustable parameter - Skyrme constant $e\left(F_{\pi}=186 \mathrm{Mev}\right.$ - experimentally measured value) are shown as variants A and B. The values of mass of $\Theta^{+}$ obtained in this way are close to the observed mass. The values of $\left\langle s_{\nu}^{2}\right\rangle$ become lower when configuration mixing takes place, and equidistant spacing of components inside of antidecuplet is violated. For the decuplet of baryons the total mass splitting increases when mixing is included, but results obtained mimic approximately the equidistant position of its components, see also discussion in the next section.

It should be stressed here that the chiral soliton approach in its present state can describe the differences of baryon or multibaryon masses [84, 42, 45]. The absolute values of mass are controlled by loop corrections of the order of $N_{C}^{0} \sim 1$ which are estimated now for the case of $B=1$ only [85]. Therefore, the value of nucleon mass in Table 2 is taken to be equal to the observed value.

As it can be seen from Table 2, the agreement with data for pure Skyrme model with one parameter is not perfect, but the observed mass of $\Theta^{+}$is reproduced with some reservation. To get more reliable predictions for masses of other exotic states the more

\footnotetext{
${ }^{6}$ The $\nu$-dependent wave functions in $S U(3)$ configuration space are simple in some cases: e.g. for $\Theta^{+}$hyperon $\Psi_{\Theta}(\nu)=\sqrt{15} \sin \nu \cos ^{2} \nu$, for the hyperon $\Omega \in\{10\}, \Psi_{\Omega}=\sqrt{5 / 2} \sin ^{3} \nu$. They are normalized according to $\int\left|\Psi_{B}(\nu)\right|^{2} 4 \sin ^{3} \nu \cos \nu d \nu=1$. In most cases the $\nu$-dependent contributions into $\left|\Psi_{B}\right|^{2}$ consist of several terms.
} 
phenomenological approach was used in [45] where the observed value $M_{\Theta}=1.54 \mathrm{GeV}$ was included into the fit, and $\Theta_{K}, \Gamma_{S B}$ were the variated parameters (variant $\mathrm{C}$ in Table 2 ). The position of some components of $\{27\},\{35\}$ and $\{\overline{35}\}$-plets is shown in Table 2 as well (they are components with largest isospin).

\begin{tabular}{|l|l|l|l|l|l|}
\hline & & $\mathrm{A}$ & $\mathrm{B}$ & $\mathrm{C}$ & \\
\hline$\Theta_{\pi}\left(\mathrm{GeV}^{-1}\right)$ & - & 6.175 & 5.556 & 5.61 & - \\
$\Theta_{K}\left(G e V^{-1}\right)$ & - & 2.924 & 2.641 & 2.84 & - \\
$\Gamma_{S B}(G e V)$ & - & 1.391 & 1.274 & 1.45 & - \\
\hline \hline Baryon $\mid N, Y, I, J>$ & $<s_{\nu}^{2}>_{0}$ & $\mathrm{~A}$ & $\mathrm{~B}$ & $\mathrm{C}$ & Data \\
\hline$N \mid 8,1, \frac{1}{2}, \frac{1}{2}>$ input & 0.467 & 939 & 939 & 939 & 939 \\
$\Lambda \mid 8,0,0,1 / 2>$ & 0.600 & 1097 & 1082 & 1103 & 1116 \\
$\Sigma \mid 8,0,1,1 / 2>$ & 0.733 & 1205 & 1187 & 1216 & 1193 \\
$\Xi \mid 8,-1,1 / 2,1 / 2>$ & 0.800 & 1316 & 1282 & 1332 & 1318 \\
\hline$\Delta \mid 10,1,3 / 2,3 / 2>$ & 0.583 & 1228 & 1258 & 1253 & 1232 \\
$\Sigma^{*} \mid 10,0,1,3 / 2>$ & 0.667 & 1359 & 1376 & 1391 & 1385 \\
$\Xi^{*} \mid 10,-1,1 / 2,3 / 2>$ & 0.750 & 1488 & 1489 & 1525 & 1530 \\
$\Omega \mid 10,-2,0,3 / 2>$ & 0.833 & 1611 & 1596 & 1654 & 1672 \\
\hline$\Theta^{+} \mid \overline{10}, 2,0,1 / 2>$ & 0.500 & 1521 & 1566 & 1539 & 1540 \\
$N^{*} \mid \overline{10}, 1,1 / 2,1 / 2>$ & 0.583 & 1637 & 1669 & 1661 & $1675 ?$ \\
$\Sigma^{*} \mid \overline{10}, 0,1,1 / 2>$ & 0.667 & 1736 & 1756 & 1764 & $1770 ?$ \\
$\Xi_{3 / 2} \mid \overline{10},-1,3 / 2,1 / 2>$ & 0.750 & 1758 & 1787 & 1786 & $1862 ?$ \\
\hline \hline$\Theta_{1}^{*} \mid 27,2,1,3 / 2>$ & 0.571 & 1648 & 1700 & 1688 & \\
$\Delta_{1}^{*} \mid 27,1,3 / 2,3 / 2>$ & 0.589 & 1780 & 1809 & 1826 & \\
$\Sigma_{2}^{*} \mid 27,0,2,3 / 2>$ & 0.607 & 1677 & 1728 & 1718 & \\
$\Xi_{3 / 2}^{*} \mid 27,-1,3 / 2,3 / 2>$ & 0.714 & 1803 & 1842 & 1850 & $1862 ?$ \\
$\Omega_{1}^{*} \mid 27,-2,1,3 / 2>$ & 0.821 & 1935 & 1959 & 1987 & \\
\hline \hline$\Theta_{2}^{*} \mid 35,2,2,5 / 2>$ & 0.708 & 1982 & 2060 & 2061 & \\
$\Delta_{5 / 2} \mid 35,1,5 / 2,5 / 2>$ & 0.438 & 1723 & 1816 & 1792 & \\
$\Sigma_{2}^{*} \mid 35,0,2,5 / 2>$ & 0.542 & 1844 & 1926 & 1918 & \\
$\Xi_{3 / 2}^{*} \mid 35,-1,3 / 2,5 / 2>$ & 0.646 & 1967 & 2037 & 2046 & \\
$\Omega_{1}^{*} \mid 35,-2,1,5 / 2>$ & 0.750 & 2091 & 2149 & 2175 & \\
$\Gamma \mid 35,-3,1 / 2,5 / 2>$ & 0.854 & 2216 & 2261 & 2306 & \\
\hline \hline $\mid \overline{35}, 3,1 / 2,3 / 2>$ & 0.562 & 2350 & 2470 & 2412 & \\
$\mid \overline{35}, 2,1,3 / 2>$ & 0.583 & 2403 & 2513 & 2466 & \\
$\mid \overline{35}, 1,3 / 2,3 / 2>$ & 0.604 & 2435 & 2541 & 2501 & \\
$\mid \overline{35}, 0,2,3 / 2>$ & 0.625 & 2437 & 2546 & 2502 & \\
$\mid \overline{35},-1,5 / 2,3 / 2>$ & 0.646 & 2417 & 2534 & 2480 & \\
$\mid \overline{35},-2,2,3 / 2>$ & 0.792 & 2573 & 2677 & 2643 & \\
\hline
\end{tabular}

Table 2. Values of masses of the octet, decuplet, antidecuplet, manifestly exotic components of higher multiplets, and highest isospin components of the $\{\overline{35}\}$-plet $(m=2)$. A: $e=3.96$; B: $e=4.12$; C: fit with parameters $\Theta_{K}, \Theta_{\pi}$ and $\Gamma_{S B}$ [45], which are shown in the upper 3 lines.

The experimental value of the mass of the $N^{*}$ candidate for antidecuplet, $M_{N^{*}}=$ $1675 \mathrm{MeV}$, is taken from recent work [81], the value $M_{N^{*}} \simeq 1680 \mathrm{MeV}$ was obtained somewhat earlier [82] in the modified partial wave anlysis of existing data. The accuracy of numerical calculations of masses given in Table 2 is not better than $\sim 1 \%$, and several 
figures are presented for convenience of different variants comparison. Small correction of numbers for variants $\mathrm{A}$ and $\mathrm{B}$ is made, in comparison with [65]. The accuracy of method itself is difficult to estimate, comparison with other quantization schemes can be useful for this. The unexpected at first sight fact that the state $\Theta^{+} \in\{\overline{10}\}$ containing strange antiquark is lighter than nonstrange component of antidecuplet, $N^{*}(I=1 / 2)$ can be easily to understood if we recall that all antidecuplet components contain $q \bar{q}$ pair: $\Theta^{+}$ contains 4 light quarks and $\bar{s}, N^{*}$ contains 3 light quarks and $s \bar{s}$ pair with some weight, $\Sigma^{*} \in\{\overline{10}\}$ contains $u, d, s$ quarks and $s \bar{s}$, etc. , see Section 7 .

The mass splitting inside of decuplet is influenced essentially by its mixing with $\{27\}$ plet components [45], see Figure, which increases this splitting considerably - the effect ignored in [43]. As a result of this mixing, the lowest in energy state of $\{10\}, \Delta$-isobar, moves considerably towards lower mass, the whole mass splitting within decuplet increases from $\sim 270 \mathrm{MeV}$ to $350-400 \mathrm{MeV}$, but equidistant position of states remains, roughly, since $\Sigma^{*}$ and $\Xi^{*}$ are mixed as well. The approximate equidistant position of the components of decuplet is not an argument against important role of mixing with other multiplets, as it is stated sometimes in literature.

The mixing of antidecuplet with the octet of baryons has considerable effect on the position of $N^{*}$ and $\Sigma^{*}(\overline{10})$ - their masses increase, the position of $\Xi_{3 / 2}^{*}$ is influenced by mixing with $\{27\}$-plet, $(J=1 / 2)$, and $\{\overline{35}\}$-plet, and its mass decreases. As a result of mixing, the total mass splitting of antidecuplet decreases slightly, opposite to the case of decuplet, and equidistant position of its components is perturbed. Position of $\Theta^{*} \in\{27\}$ is influenced by mixing with higher multiplets [45], the components of $\{35\}$-plet mix mainly with corresponding components of septuquark $\{64\}$-plet.

The mass of $\Theta$ hyperon is obtained in the interval $1520-1560 \mathrm{MeV}$ for the Skyrme parameter $e$ between 3.96 and 4.12, in the rigid rotator approximation, so the statement made in [59] that "all models appear to normalise to some feature and do not naturally explain the low mass of the orbitally excited pentaquark" does not apply to the simple chiral soliton model which is $S U(3)$ generalization of the original Skyrme model quantized as rigid rotator.

The flavor symmetry breaking in the kaon decay constant, i.e. the fact that $F_{K} / F_{\pi}=$ 1.22 leads to certain increase of the kaonic moment of inertia and to decrease of the $\Theta$ mass [45]

$$
\Theta_{K}=\frac{1}{8} \int\left(1-c_{f}\right)\left[F_{K}^{2}-\left(F_{K}^{2}-F_{\pi}^{2}\right) \frac{2-c_{f}}{2} s_{\nu}^{2}+\frac{1}{e^{2}}\left(f^{\prime 2}+\frac{2 s_{f}^{2}}{r^{2}}\right)\right] d^{3} r
$$

where $f(r)$ is the profile function of skyrmion, $f(0)=\pi$ at the origin, and $f(\infty)=0$. This moment of inertia is maximal when the angle of rotation into strange direction $\nu=0$, see Table 2, and this corresponds to rigid rotor approximation used previously and in [45]. $\Theta_{K}$ decreases when $\nu$ deviates from 0 , and indeed, the masses of exotic baryons obtained within soft, or slow rotator approximation, are considerably greater [86].

It should be noted that predictions of the mass of $\Xi_{3 / 2}^{*}$ made in [45] half a year before its observation at CERN [21] were quite close to the reported value $1862 \mathrm{MeV}$ : it was $1786 \mathrm{MeV}$ for the component of antidecuplet, and $1850 \mathrm{MeV}$ for the $\{27\}$ component, variant $\mathrm{C}$ of Table 2. Predictions for masses of cryptoexotic components of $\{\overline{10}\}$ and $\{27\}$-plet are clear from Table 2 as well.

It was stated in the paper [55] that the spectrum of antidecuplet obtained "from correlated quark picture differs in several dramatic ways from the spectrum predicted by the chiral soliton model", and "the prediction of light charged exotic $\Xi$ 's is the most distinctive signature of our model". The following comment is necessary here. Indeed, it was apparent contradiction of estimates made in [55] and results obtained in [43] within particular variant of chiral quark-soliton model where the total splitting of antidecuplet 
was found equal to $540 \mathrm{MeV}^{7}$. As shown in [45] and above, the total splitting of anti-10 in Skyrme-type model equals to $\Delta_{M}(\overline{10})=1.5 \Gamma_{S B} \Delta_{S C}(\overline{10}) \simeq 270 \mathrm{MeV}$ if configuration mixing is not included, and less than $\sim 270 \mathrm{MeV}$, about $250 \mathrm{MeV}$ if mixing is taken into account. $\Delta_{S C}(\overline{10})=1 / 8$ is the splitting of strangeness contents within antidecuplet, which can be expressed in terms of Clebsch-Gordan coefficients of the $S U(3)$ group. Therefore, there is no such dramatic difference between simplified quark model picture of [55] and chiral soliton model predictions, as it was claimed in [55], but results of [45], available since April 2004, have not been considered in [55]. Another comment is that picture of ideal mixing between octet and antidecuplet of pentaquark states, proposed in [55], can be disturbed by mixing with ground state octet, as it takes place in chiral soliton approach $[45,53]$. Normally, the ground state octet contains admixture of antidecuplet (about $(5-6) \%$ in probability for the nucleon, variant $\mathrm{C}$ of Table 2$),\{27\}$-plet $(\sim 3 \%)$, and smaller amounts of higher multiplets, since the number of quark-antiquark pairs is not conserved by strong interactions. Further developments of the correlated quark models with diquarks or triquarks are of interest, also if the announced pentaquark states are not confirmed.

In Table 3 predictions of the mass of exotic $\Phi / \Xi_{3 / 2}$ state with strangeness $S=-2$ which were made before experimental evidence for this state [21], are presented. The paper [47] repeated the approach of [43], the mass of $\Xi_{3 / 2} \in\{27\}$ shown in Table 3 was a new result in comparison with [43]. The state observed in [21] could belong just to $\{27\}$-plet, another doubly strange state from anti-10 should exist in this case, with smaller mass and more narrow than the observed one. Of course, this discussion becomes irrelevant if the $\Phi / \Xi_{3 / 2}$ state is not confirmed.

\begin{tabular}{|c|l|l|l|l|l|l|}
\hline & DPP $[43]$ & WK[45] & JW[55] & BFK[47] & P[46] & Datum[21] \\
\hline$\Phi / \Xi_{3 / 2} \in\{\overline{10}\}$ & 2070 & $1780-1790$ & 1750 & - & 1800 & 1862 \\
$\Xi_{3 / 2} \in\{27\}$ & - & 1850 & - & 2048 & - & $1862 ?$ \\
\hline
\end{tabular}

Table 3. Predictions of the mass of doubly strange hyperon $\Phi / \Xi_{3 / 2}$ in chronological order. The value by M.Praszalowicz, $1800 \mathrm{MeV}$, is taken from figure in [46] and is approximate by this reason.

The component of $\{35\}$-plet with zero strangeness and $I=J=5 / 2$ is of special interest because it has the smallest strangeness content (or $s_{\nu}^{2}$ ) - smaller than nucleon and $\Delta$, see Table 2. It is the lightest component of $\{35\}$-plet, and this remarkable property has explanation in simplistic pentaquark model, see Section 7 below. As a consequence of isospin conservation by strong interactions it can decay into $\Delta \pi$, but not to $N \pi$ or $N \rho$.

Generally, the baryon resonances which belong to $\{35\}$-plet cannot be obtained in meson-baryon interactions, or in some decay into meson (from the octet) and baryon, also from octet, due to $S U(3)$ invariance of strong interactions, see also [18]. The components of $\{35\}$ with highest isospin which are manifestly exotic, cannot be formed in this way also due to isospin invariance of strong interactions, and this is essentially more rigorous prohibition.

The masses of lowest $m=2$ multiplet, $\{\overline{35}\}$-plet, are shown in Table 2 as an example: this state is not a pentaquark, but septuquark (or heptaquark). The partners of antidecuplet with spin $J=3 / 2$ are contained within this multiplet, see section 8 .

\footnotetext{
${ }^{7}$ Later, when observation of the resonance $\Xi^{--}$has been reported in [21], the authors [43] revised their result in [79] and identified the lowest and the highest masses of antidecuplet with observed values, i.e. they put the total mass splitting of $\overline{10}$ equal to the value $\sim 324 \mathrm{MeV}$ taken from experiment. Cryptoexotic components of antidecuplet, $N^{*}$ and $\Sigma^{*}$, have been placed within this mass gap. The change of the experimental value of the pion-nucleon sigma-term was important for this re-analysis, the latest and largest value of sigma-term was obtained in [80].
} 


\subsection{Model of the slow (soft) rotator}

An alternative method of calculation is the soft (or slow) rotator approximation developed for the case of $B=1$ by Schwesinger and Weigel [84], and used in [86] to describe the strange dibaryons spectrum. It is supposed within this approximation that for each value of the angle $\nu$ it is sufficient time to rearrange the profile function under influence of forces due to flavor symmetry breaking terms in the Lagrangian. Estimates show that for $B=1$ the rigid rotator approximation is better, whereas for $B \geq 2$ the soft rotator becomes more preferable. Indeed, the rotation time in cofiguration space can be estimated as $\tau_{\text {rot }} \sim \pi / \omega$, and the angular velocity $\omega \sim \sqrt{C_{2}(S U 3)} / \Theta_{K}$ for definite $S U(3)$ multiplet, see Table 1. It is difficult to estimate the time necessary for rearranging the profile function under influence of FSB forces, one can state only that it is greater than time necessary for signal propagation accross skyrmion, $\tau_{\text {sign }} \sim 2 R_{S}$. So, we come to the inequality $\pi \Theta_{K} \ll 2 R_{S} \sqrt{C_{2}(S U 3)}$ which should be fulfilled for the rigid rotator approximation being valid. For $B=1$ both sides of this inequality are of the same order of magnitude, although left side is somewhat smaller.

\begin{tabular}{|l|l|r|r|}
\hline \hline Baryon $|N, Y, I, J\rangle$ & $\left\langle s_{\nu}^{2}>\right.$ & \multicolumn{1}{|c|}{ SR } & Data \\
\hline$N \mid 8,1, \frac{1}{2}, \frac{1}{2}>$ input & 0.314 & 939 & 939 \\
$\Lambda \mid 8,0,0,1 / 2>$ & 0.500 & 1110 & 1116 \\
$\Sigma \mid 8,0,1,1 / 2>$ & 0.602 & 1220 & 1193 \\
$\Xi \mid 8,-1,1 / 2,1 / 2>$ & 0.740 & 1320 & 1318 \\
\hline$\Delta \mid 10,1,3 / 2,3 / 2>$ & 0.315 & 1240 & 1232 \\
$\Sigma^{*} \mid 10,0,1,3 / 2>$ & 0.483 & 1415 & 1385 \\
$\Xi^{*} \mid 10,-1,1 / 2,3 / 2>$ & 0.650 & 1560 & 1530 \\
$\Omega \mid 10,-2,0,3 / 2>$ & 0.790 & 1670 & 1672 \\
\hline$\Theta \mid \overline{10}, 2,0,1 / 2>$ & 0.380 & 1737 & 1540 \\
$\Xi_{3 / 2} \mid \overline{10},-1,3 / 2,1 / 2>$ & 0.607 & 2118 & $1862 ?$ \\
\hline \hline$\Theta_{1}^{*} \mid 27,2,1,3 / 2>$ & 0.416 & 1840 & \\
$\Sigma_{2}^{*} \mid 27,0,2,3 / 2>$ & 0.438 & 1880 & \\
$\Xi_{3 / 2}^{*} \mid 27,-1,3 / 2,3 / 2>$ & 0.594 & 2090 & \\
$\Omega_{1}^{*} \mid 27,-2,1,3 / 2>$ & 0.755 & 2270 & \\
\hline \hline$\Theta_{2}^{*} \mid 35,2,2,5 / 2>$ & 0.464 & 2180 & \\
$\Delta_{5 / 2} \mid 35,1,5 / 2,5 / 2>$ & 0.242 & 1750 & \\
$\Sigma_{2}^{*} \mid 35,0,2,5 / 2>$ & 0.382 & 1990 & \\
$\Xi_{3 / 2}^{*} \mid 35,-1,3 / 2,5 / 2>$ & 0.528 & 2190 & \\
$\Omega_{1}^{*} \mid 35,-2,1,5 / 2>$ & 0.675 & 2370 & \\
$\Gamma \mid 35,-3,1 / 2,5 / 2>$ & 0.854 & 2530 & \\
\hline \hline $\mid \overline{35}, 3,1 / 2,3 / 2>$ & 0.442 & 2900 & \\
$\mid \overline{35},-1,5 / 2,3 / 2>$ & 0.477 & 3030 & \\
$\mid \overline{35},-2,2,3 / 2>$ & 0.641 & 3385 & \\
\hline
\end{tabular}

Table 4. Values of masses of the octet, decuplet, antidecuplet and manifestly exotic components of higher multiplets within soft rotator (SR) approximation [84]: $e=3.46, F_{K} / F_{\pi}=1.26$, the values of $\left\langle s_{\nu}^{2}\right\rangle$ are calculated with configuration mixing included. 
Thus we see, that rigid rotator is really more preferable for $B=1$, especially for exotic multiplets (since the value of $C_{2}(S U 3)$ is greater, see Table 1$)^{8}$. With increasing baryon number left side of this inequality grows faster than right side, therefore slow rotator approximation may become better for greater baryon numbers. In view of conflicting experimental situation on pentaquarks observation it makes sense to present the results for pentaquarks spectra within this approximation as well.

A natural thing is that the masses of exotic states within soft rotator model are greater than in the rigid rotor approximation: the strange, or kaonic inertia $\Theta_{K}$ becomes smaller for slow rotator, as explained above, (17). However, it looks somewhat unexpected that increase of masses is so great, up to $\sim 200 \mathrm{MeV}$. Anyway, for $B=1$ the rigid rotator approximation is better justified [65]. Strangeness contents of baryon states shown in Table 4 are calculated with configuration mixing included, and they are considerably smaller than SC calculated in first order of perturbation theory, Table 2. The slow rotator approximation [84] deserves more attention if the negative results on $\Theta$ observation are confirmed.

All baryonic states considered here are obtained by means of quantization of soliton rotations in $S U(3)$ configuration space, therefore they have positive parity. A qualitative discussion of the influence of other (nonzero) modes - vibration, breathing - as well as references to corresponding papers can be found in $[44,45]$. Calculation of baryon spectra with monopole excitation is made in $[44,51]$. The realistic situation can be more complicated than somewhat simplified picture presented here, since each rotation state can have vibrational excitations with characteristic energy of hundreds of $M e V$. The resonance $N^{*}(1440)$ is just the monopole excitation of ground state nucleon [44, 51].

\section{Comments on the $\Theta$ width and large $N_{c}$ arguments}

If the matrix element of the decay $\Theta^{+} \rightarrow K N$ is written in a form

$$
M_{\Theta \rightarrow K N}=g_{\Theta K N} \bar{u}_{N} \gamma_{5} u_{\Theta} \phi_{K}^{\dagger}
$$

with $u_{N}$ and $u_{\Theta}$ - bispinors of final and initial baryons, then the decay width equals to

$$
\Gamma_{\Theta \rightarrow K N}=\frac{g_{\Theta K N}^{2}}{8 \pi} \frac{\Delta_{M}^{2}-m_{K}^{2}}{M^{2}} p_{K}^{c m} \simeq \frac{g_{\Theta K N}^{2}}{8 \pi} \frac{\left(p_{K}^{c m}\right)^{3}}{M m_{N}}
$$

where $\Delta_{M}=M-m_{N}, M$ is the mass of decaying baryon, $p_{K}^{c m} \simeq 269 \mathrm{MeV} / \mathrm{c}$ if $M_{\Theta}=$ $1540 \mathrm{MeV}$ - the kaon momentum in the c.m. frame. For the decay constant we obtain then $g_{\Theta K N} \simeq 4.4$ if we take the value $\Gamma_{\Theta \rightarrow K N}=10 \mathrm{MeV}$ as suggested by experimental data [3],[25]. This should be compared with pion-nucleon coupling constant $g_{\pi N N} \simeq 13.14$ (according to latest analysis [83] $\left.g_{\pi N N}^{2} /(4 \pi)=13.75 \pm 0.10\right)$. So, suppression of the decay $\Theta \rightarrow K N$ takes place, but not very large if the width is really close to $10 \mathrm{MeV}$.

Prediction of the widths of baryon resonances is not an intrinsic property of the chiral soliton approach - in distinction from spectra of states. Additional assumptions concerning the form of transition amplitudes are necessary $[43,46]$. Numerical cancellation in the matrix element of $\Theta$ decay was obtained in [43], and later proved also in large $N_{c}$ limit in chiral quark soliton model, for vanishing dimension of the soliton [46]. It would be difficult, however, to explain the width $\Gamma_{\Theta} \sim 1 \mathrm{MeV}$ or smaller, as suggested by scattering data $[14,15]$.

\footnotetext{
${ }^{8}$ If we take into account connection between soliton radius and another (isotopic) moment of inertia, $\Theta_{I} \sim M_{S} R_{S}^{2} / 2$, this condition will take the form $\Theta_{K}<\sqrt{\Theta_{I} C_{2}(S U 3) / M_{S}}$.
} 
The arguments have been presented in the literature [66], see also [46], that in large $N_{c}$ limit and in the case of chiral symmetry, i.e. when $m_{\pi}=m_{K}=0$, one should expect that $\Theta$ width is parametrically greater than width of $\Delta(1232)$ isobar, in contradistinction from what is seen experimentally. As it has been observed long ago, the mass splitting between antidecuplet and octet of baryons is of the order of $N_{c}^{0} \sim 1$, whereas that between decuplet and octet is of the order of $N_{c}^{-1}$ due to the difference in rotation energy. To make these conclusions, the identification of multiplets in our $N_{c}=3$ world and hypothetical large $N_{c}$ world is made in definite way, and this identification is not unique in the latter case.

It is known that the artifact of large $N_{c}$ generalization of the chiral soliton and the quark models is appearance of multiplets of baryons which are absent in real $N_{c}=3$ world ${ }^{9}$. For nonexotic baryons there are $\left(N_{c}+1\right) / 2 S U(3)$ multiplets, beginning with $[p, q]=\left[1,\left(N_{c}-1\right) / 2\right]$ which is interpreted as analogue of $N_{c}=3$ octet. The next one is the multiplet with $(p, q)=\left[3,\left(N_{c}-3\right) / 2\right]$ interpreted as "decuplet", and the multiplet with largest $p$ is that with $(p, q)=\left(N_{c}, 0\right)$. All multiplets except first two are usually ignored, even not mentioned. To discuss the large $N_{c}$ properties of any particular state, one should first establish correspondence between such state in real world and in miraculous large $N_{c}$ world, and the way to do this depends on the principle which is taken as leading one. The $Y=N_{c} / 3$ state within $\left(1,\left(N_{c}-1\right) / 2\right)$ multiplet not only has the minimal possible for any baryon isospin (and spin) $I=1 / 2$, but is also the state maximally antisymmetrized in isospin and spin variables. It is natural to consider it as analogue of nucleon by this reason as well.

The state with $Y=1$ from $(3,0)$ multiplet in $N_{c}=3$ world not only is a state with isospin $I=3 / 2$, but it is also a state of maximal symmetry in isospin and spin variables. So, if we take this principle of maximal symmetry as a leading one, we should take the state with $Y=N_{c} / 3, I=N_{c} / 2$ from the multiplet $(p, q)=\left(N_{c}, 0\right)$ as analogue of $\Delta$ in the large $N_{c}$ world. The rotation energy of this state quantized as rigid rotor is very large, $N_{c}\left(N_{c}+2\right) /\left(8 \Theta_{I}\right) \sim N_{c}$, leading to parametrically large width of " $\Delta$ " baryon. The ratio of " $\Delta "$ " $N "$ mass splitting to " $\Theta "-" N "$ splitting is of the order $\sim N_{c}$ in this case. Even if not quite convincing, this example shows that large $N_{c}$ argumentation is not without ambiguity because identification of baryons of $N_{c}=3$ and large $N_{c}$ worlds is a subtle question.

The difference of masses of particular baryons, e.g. of $\Delta \in\{10\}$ and nucleon from ground state octet contains also some contribution due to $F S B$ terms and different values of their strangeness content. Strangeness content of the nucleon is $S C_{N}=7 / 30$, and $S C_{\Delta}=7 / 24$, see Table 2 and formula (14). Strangeness contents of analogues of nucleon in $\left[1,\left(N_{c}-1\right) / 2\right]$ and " $\Delta "$ in $\left[3,\left(N_{c}-3\right) / 2\right]$ multiplets are given in Appendix. Their difference is $S C_{\Delta}-S C_{N}=2\left(N_{c}+4\right)\left[1 /\left[\left(N_{c}+1\right)\left(N_{c}+9\right)\right]-1 /\left[\left(N_{c}+3\right)\left(N_{c}+7\right)\right]\right]$. At large $N_{c}$ this difference decreases like $1 / N_{c}^{3}$, therefore it gives negligible contribution to the baryons mass differences.

In reality the masses of $\pi$-meson and especially of kaons are not only different from zero, but even comparable with mass splittings - both masses are formally of the order of $N_{c}^{0} \sim 1$. The width of $\Theta$ depends on result of cancellation of two quantities, each of them is of the order of $N_{c}^{0} \sim 1$ : " $\Theta-N$ " mass splitting and kaon mass. For the case of $\Theta \rightarrow K N$ almost all energy release is absorbed by the kaon mass. Therefore, the phase space suppression of the $\Theta$ decay cannot be controlled by $1 / N_{c}$ counting arguments only, since finally it depends on subtraction of two quantities of same order of magnitude, $N_{c}^{0} \sim 1$, but different nature, at least in our present understanding. The result of this subtraction looks occasional, it could be even negative, thus making the $\Theta$ baryon stable

\footnotetext{
${ }^{9}$ This is not the only problem. The hypercharge for arbitrary (but odd) $N_{c}$ is $Y=N_{c} B / 3+S$ ([39], see also $[68,66])$, and the electric charge defined by relation $Q=I_{3}+Y / 2$ is integer only if $N_{c}$ is multiple of 3. Another possibility for electric charges was discussed in [87], see Appendix.
} 
relative to strong interactions. There are many examples in physics when some quantities of crucial importance cannot be deduced from general principles ${ }^{10}$.

Without any doubt, the width of exotic states is extremely important and interesting quantity, especially if the width of the order of $1 \mathrm{MeV}$ for $\Theta^{+}$is confirmed. The cheking of scattering data used in the analyses of $[14,15]$ seems to be quite important, see also [18]. Critical review of these scattering data was made recently in [19] where necessity of their checking also has been emphasized.

\section{Wave functions of pentaquarks and the masses of strange quark (antiquark)}

Similar to the case of baryons and mesons made of valence quarks (antiquarks), it is convenient to discuss the properties of new baryon resonances in terms of their quark wave functions (WF). The question about correspondence of chiral soliton model results and expectations from the quark models is quite interesting and even thorny.

\subsection{Quark contents of pentaquarks}

The quark contents of wave functions of manifestly exotic resonances are unique within pentaquark approximation, i.e. the number of quarks or antiquarks of definite flavor is fixed by their strangeness and isospin ${ }^{11}$. It is easy to obtain for WF of manifestly exotic components of antidecuplet (see also the Figure):

$$
\Psi_{\Theta} \sim u u d d \bar{s}
$$

and for 4 components of exotic $S=-2, I=3 / 2$ state

$$
\Psi_{\Phi / \Xi_{3 / 2}} \sim \operatorname{ssd} \bar{u} ; \operatorname{ssd}(u \bar{u}-d \bar{d}) / \sqrt{2} ; \operatorname{ssu}(d \bar{d}-u \bar{u}) / \sqrt{2} ; \operatorname{ssuu} \bar{d} .
$$

Quark content of cryptoexotic states WF are not unique. Within antidecuplet:

$$
\begin{gathered}
\Psi_{N^{*}} \sim u d d\left[\alpha_{-} u \bar{u}+\beta_{-} d \bar{d}+\gamma_{-} s \bar{s}\right] ; \quad u u d\left[\alpha_{+} u \bar{u}+\beta_{+} d \bar{d}+\gamma_{+} s \bar{s}\right], \\
\Psi_{\Sigma^{*}} \sim s d d\left[\mu_{-} u \bar{u}+\nu_{-} d \bar{d}+\rho_{-} s \bar{s}\right] ; \ldots ; s d d\left[\mu_{+} u \bar{u}+\nu_{+} d \bar{d}+\rho_{+} s \bar{s}\right],
\end{gathered}
$$

coefficients $\alpha_{-}, \alpha_{+}$, etc. depend on the particular variant of the model.

E.g., for the model with diquark transforming like flavor anti-triplet, $D_{q} \sim \overline{3}_{F}[55]$, $\alpha_{-}=\sqrt{1 / 3}, \beta_{-}=0, \gamma_{-}=\sqrt{2 / 3}$, etc. Equidistancy within $\overline{10}$ was obtained in $[56,57]$ for this case.

Within $\{27\}$-plet only the $S=0, I=3 / 2$-state (analogue of $\Delta$-isobar) is cryptoexotic. The states with $S=+1, I=1$ and state with $S=-1, I=2$ contain one $s$-quark field as depicted in the Figure, and their masses do not differ much by this reason, as it was obtained in chiral soliton model as well, see Table 2.

Within $\{35\}$-plet all states of maximal isospin are manifestly exotic and have unique quark content. The state with $S=0, I=5 / 2$ (it can be called $\Delta_{5 / 2}$ ) does not contain strange quarks:

$$
\Psi_{\Delta_{5 / 2}} \sim d d d d \bar{u} ; \ldots ; \text { uuuu } \bar{d}
$$

\footnotetext{
${ }^{10}$ The particular values of binding energies of nuclei or nuclear levels responsible for stability or instability of nuclear isotopes, and for spectra of photons and neutrinos emitted by stars could be one of such examples.

${ }^{11}$ For pentaquarks manifestly exotic states are defined by relation $I=(5+S) / 2$ if strangeness $S \leq 0$ (any state with $S>0$ is manifestly exotic, as discussed in Introduction).
} 
neither $s$, nor $\bar{s}$ quarks! Remarkably, that within chiral soliton model this state has minimal, among all baryons, strangeness content $\left(S C\left(\Delta_{5 / 2}\right) \simeq 0.22\right)$, and has the lowest (within $\{35\}$-plet) mass, see Table 2 and [45].

Evidently, besides flavor antitriplet diquark $D_{q} \sim \overline{3}_{F}$ (anti-triplet in color, singlet $L=0$ state) discussed in this context in [55] and called also "good" diquark [58], the diquarks $D_{q} \sim 6_{F}$ ("bad" diquarks, transforming also like $\overline{3}$ in color, triplet $L=0$ states) are necessary to form $\{27\}$ - and $\{35\}$-plets of pentaquarks.

Let us denote $\left(q_{1} q_{2}\right)$ the flavor symmetric diquark with spin $J=1\left(\overline{3}_{C}\right.$ in color, triplet $L=0$ state). Then realization of the wave function of $\{27\}$-plet of pentaquarks via diquarks is (we use same notation $\mid N(p, q), Y, I, I_{3}>$ for the components of multiplets as in Table $\mathbf{2}$, and present the states with lowest value of $I_{3}$ ):

$$
\Psi_{\mid 27,2,1,-1>} \sim\left(d_{1} d_{2}\right)\left[d_{3} u_{4}\right] \bar{s},
$$

with $\left[u_{3} d_{4}\right]=\left(u_{3} d_{4}-d_{3} u_{4}\right) / \sqrt{2}$,

$$
\Psi_{\mid 27,1,3 / 2,-3 / 2>} \sim\left[-\left(d_{1} d_{2}\right)\left[u_{3} d_{4}\right] \bar{u}+\left(d_{1} d_{2}\right)\left[s_{3} d_{4}\right] \bar{s}\right] / \sqrt{2},
$$

other components of this $Y=1$ isomultiplet can be obtained easily with the help of isospin raising $I^{+}$operator. States with negative strangeness have the wave functions

$$
\begin{aligned}
\Psi_{\mid 27,0,2,-2>} & \sim\left(d_{1} d_{2}\right)\left[s_{3} d_{4}\right] \bar{u}, \\
\Psi_{\mid 27,-1,3 / 2,-3 / 2>} & \sim\left(d_{1} s_{2}\right)\left[s_{3} d_{4}\right] \bar{u}, \\
\Psi_{\mid 27,-2,1,-1>} & \sim\left(s_{1} s_{2}\right)\left[s_{3} d_{4}\right] \bar{u} .
\end{aligned}
$$

The components with other projections of isospin $I_{3}$ are not shown here since they can be obtained easily.

For the $\{35\}$-plet two flavor-symmetric diquarks $D_{6 F}$ are necessary to form the states with maximal isospin, according to group theoretical equality $6 \otimes 6 \otimes \overline{3}=\{35\} \oplus\{27\} \oplus$ $2\{10\} \oplus\{\overline{10}\} \oplus 2\{8\}$. For example, the $S=+1 \Theta^{*}$ state has wave function

$$
\Psi_{\mid 35,2,2,-2>} \sim\left(d_{1} d_{2}\right)\left(d_{3} d_{4}\right) \bar{s},
$$

the above mentioned $\Delta_{5 / 2}$ has

$$
\Psi_{\mid 35,1,5 / 2,-5 / 2>} \sim\left(d_{1} d_{2}\right)\left(d_{3} d_{4}\right) \bar{u}
$$

etc. States with other isospin projections also can be obtained easily. The antidecuplet can be made from two symmetric diquarks $D_{6 F}$ as well, e.g. its $S=+1$ component made of two isovector diquarks is

$$
\Psi_{\Theta^{+}} \sim\left[u_{1} u_{2} d_{3} d_{4}+d_{1} d_{2} u_{3} u_{4}-\frac{1}{2}\left(u_{1} d_{2}+u_{2} d_{1}\right)\left(u_{3} d_{4}+d_{3} u_{4}\right)\right] \bar{s}
$$

it is expected to have considerably higher energy [58].

\subsection{Mass of strange antiquark in different pentaquark multiplets}

Here we shall compare the mass spectrum of baryons obtained within chiral soliton model (CSM) with the quark model in pentaquark approximation, which will allow to make some conclusions concerning masses of strange quarks, antiquarks and diquarks, necessary to fit the chiral soliton model predictions. The contribution of strange quark mass $\left(m_{s}\right)$, 
antiquark mass $\left(m_{\bar{s}}\right)$ and strange diquark mass $\left(m_{s \bar{s}}\right)$ to masses of pentaquark states is presented in Table 5 , in the lines below notations of states.

It is easy to see that without configuration mixing (the first lines of numbers in Table 5) there is linear dependence of masses on hypercharge of states for antidecuplet; for $\{27\}$-plet the states with hypercharges $Y=2,1,0$ belong to one line, and states with $Y=0,-1,-2$ - to another line; for $\{35\}$-plet 5 states with hypercharge from $Y=1$ down to $Y=-3$ are on one line. Such linear dependence, however, is not specific for CSM only, but is the consequence of the special way of $S U(3)$-symmetry breaking, when FSB terms in lagrangian are proportional to the $D_{88}$ Wigner function, or to hypercharge, which leads to the Gell-Mann - Okubo formula

$$
\Delta M_{F S B}=a\left[Y^{2} / 4-I(I+1)\right]+b Y,
$$

with $a, b$ - some constants different for different $S U(3)$ multiplets.

For antidecuplet the relation between hypercharge and isospin takes place $I=1-Y / 2$. For $\{27\}$-plet the states $|Y, I>=| 0,2>, \mid-1,3 / 2>$ and $\mid-2,1>$ are on the line $I=Y / 2+2$, the states $|Y, I>=| 0,2>, \mid 1,3 / 2>$ and $\mid 2,1>$ belong to the line $I=-Y / 2+2$. For the components of $\{35\}$-plet with $Y$ from 1 to -3 similar relation takes place $I=Y / 2+2$. It is easy to see that in all these cases quadratic in $Y$ term in formula (20) cancels, and linear dependence of the mass on hypercharge takes place. Similar results have been obtained recently in [88].

Less trivial and more informative are some relations for masses of strange quarks/antiquarks which follow from comparison with the quark model. In what follows we shall reserve a possibility that effective masses of strange quark and antiquark are different, as well as they are different within different $(p, q)$ multiplets. This effect is known already since it takes place for ground states octet and decuplet of baryons as well: the effective strange quark mass is $189 \mathrm{MeV}$ within octet and $147 \mathrm{MeV}$ within decuplet, in average ${ }^{12}$.

We can easily obtain within pentaquark approximation, ascribing the mass difference of different components to the strange quark (antiquark) mass, the following relations:

$$
\Delta_{M}(\{\overline{10}\})=\left[2 m_{s}-m_{\bar{s}}\right]_{\{\overline{10}\}} .
$$

Recall that for decuplet

$$
\Delta_{M}(\{10\})=\left[3 m_{s}\right]_{\{10\}},
$$

so, in oversimplified model where $m_{s}(\{\overline{10}\})=m_{\bar{s}}(\{\overline{10}\})=m_{s}(\{10\})$ the first one should be 3 times smaller than $\Delta_{M}(\{10\})$. However, this condradiction becomes much softer and even can dissappear in more refined models where masses of strange quark and antiquark are different, as well as they are different in different $S U(3)$ multiplets.

The equality of mass differences between adjacent components of antidecuplet:

$$
\frac{2}{3} m_{s \bar{s}}-m_{\bar{s}}=m_{s}-\frac{1}{3} m_{s \bar{s}}
$$

has a consequence that the mass of $s \bar{s}$ pairs equals simply to the sum of quark and antiquark masses:

$$
m_{s \bar{s}}=m_{s}+m_{\bar{s}}
$$

the index $\overline{10}$ is omitted for all masses. Equality similar to (23) holds for masses within $\{27\}$-plet as well. Relations $(21,23)$ are the only relations which can be obtained for masses of strange quark and antiquark within antidecuplet, leaving otherwise much freedom for these masses.

\footnotetext{
${ }^{12}$ When hyperfine splitting contributions are included, this difference between strange quark masses extracted from octet and decuplet, becomes much smaller.
} 


\begin{tabular}{|l|l|l|l|l|l|}
\hline $\mid \overline{10}, 2,0>$ & $\mid \overline{10}, 1,1 / 2>$ & $\mid \overline{10}, 0,1>$ & $\mid \overline{10},-1,3 / 2>$ & & \\
\hline$m_{\bar{s}}$ & $2 m_{s \bar{s}} / 3$ & $m_{s}+m_{s \bar{s}} / 3$ & $2 m_{s}$ & & \\
\hline 564 & 655 & 745 & 836 & & \\
600 & 722 & 825 & 847 & & \\
\hline \hline $\mid 27,2,1>$ & $\mid 27,1,3 / 2>$ & $\mid 27,0,2>$ & $\mid 27,-1,3 / 2>$ & $\mid 27,-2,1>$ & \\
\hline$m_{\bar{s}}$ & $m_{s \bar{s}} / 2$ & $m_{s}$ & $2 m_{s}$ & $3 m_{s}$ & \\
\hline 733 & 753 & 772 & 889 & 1005 & \\
749 & 887 & 779 & 911 & 1048 & \\
\hline \hline $\mid 35,2,2>$ & $\mid 35,1,5 / 2>$ & $\mid 35,0,2>$ & $\mid 35,-1,3 / 2>$ & $\mid 35,-2,1>$ & $\mid 35,-3,1 / 2>$ \\
\hline$m_{\bar{s}}$ & 0 & $m_{s}$ & $2 m_{s}$ & $3 m_{s}$ & $4 m_{s}$ \\
\hline 1152 & 857 & 971 & 1084 & 1197 & 1311 \\
1122 & 853 & 979 & 1107 & 1236 & 1367 \\
\hline
\end{tabular}

Table 5. Masses of components of $\{\overline{10}\}$, and components with maximal isospin for $\{27\}, J=3 / 2$ and $\{35\}, J=5 / 2$-plets of exotic baryons (in $M e V$, the nucleon mass is subtracted). The contribution of strange quarks and antiquarks is written below notation of states, $m_{s \bar{s}}$ is the mass of the $s \bar{s}$ pair. The first line of numbers is the result of calculation without configuration mixing, the second line - configuration mixing included according to [45]. Calculations correspond to variant $C$ of Table 2, or case $A$ of paper [45]: $\Theta_{K}=2.84 \mathrm{GeV}^{-1}, \Theta_{\pi}=5.61 \mathrm{GeV}^{-1}, \Gamma=1.45 \mathrm{GeV}$.

More information can be obtained for strange quark/antiquark masses within higher exotic multiplets. Linear dependence of masses of manifestly exotic components of $\{27\}$ plet allows to obtain for the mass of strange quark $m_{s} \in\{27\} \simeq 117 \mathrm{MeV}$ (configuration mixing not included). For $\{27\}$-plet it is also useful to fix the difference of masses between manifestly exotic components $\mid 27,2,1>$ and $\mid 27,0,2>$ :

$$
\Delta_{2-0}(\{27\})=\left[m_{\bar{s}}-m_{s}\right]_{\{27\}} \simeq-40 \mathrm{MeV},
$$

so, strange antiquark within 27-plet should be lighter than strange quark, according to CSM results.

Within 35-plet, it follows from the results for masses of the components with strangeness $S \leq 0$ that the effective strange quark mass is about $115 \mathrm{MeV}$. If we ascribe the difference of masses between $Y=2$ and $Y=1$ states of $\{35\}$-plet to the mass of strange antiquark, we obtain that $m_{\bar{s}} \in\{35\} \simeq 295 \mathrm{MeV}$. Strong interactions of the quark $s$ and antiquark $\bar{s}$ are different, so no wonder that effective masses of quark and antiquark are different. However, such big difference between masses of strange antiquarks in 27 and 35-plets seems to be unexpected.

Configuration mixing increases the mass of strange quark within 27-plet up to $135 \mathrm{MeV}$. Within 35-plet configuration mixing does not change the above numbers drastically: the effective strange quark mass increases up to $125-130 \mathrm{MeV}$, and the mass of antiquark $\bar{s}$ decreases to $\sim 270 \mathrm{MeV}$.

The effect of configuration mixing is especially important for cryptoexotic components of antidecuplet $(Y=1$ and 0$)$ which mix with similar components of the lowest baryon octet, as a result, their masses increase. The $\Phi / \Xi_{3 / 2}$ component is mixed with analogous component $\Xi_{3 / 2} \in\{27\}$, and its mass moves to lower value. In summary, after mixing the total mass splitting of antidecuplet decreases, and equidistant position of states is considerably violated, unlike the case of decuplet. Within 27-plet, configuration mixing increases the mass of cryptoexotic state $\mid 27,1,3 / 2>$ considerably (more than by $130 \mathrm{MeV}$ ). The identification of this state, analogue of $\Delta(1232)$-isobar, is not straihgtforward, see also recent analysis in [18].

The comparison of masses of $\Theta^{+} \in \overline{10}$ and $\Theta^{*} \in 27$ allows to conclude that $6_{F}$ diquark is heavier than $\overline{3}_{F}$ diquark by $\sim 120-150 \mathrm{MeV}$, if we ascribe the mass difference of 
$\Theta$-resonances to the mass difference of diquarks. It is possible to estimate the mass difference of diquarks more straightforward, in the limit $m_{K} \rightarrow 0$. Then we obtain $\Delta M_{(6 F-\overline{3} F)} \simeq 3 /\left(2 \Theta_{\pi}\right)-1 /\left(2 \Theta_{K}\right) \sim 100 \mathrm{MeV}$. This is smaller than the estimate given by F.Wilczek in [58], $\Delta M_{(6 F-\overline{3} F)} \sim 240-360 \mathrm{MeV}$.

The comparison of masses of $\Theta^{*} \in 27$ and $\Theta^{*} \in 35$, taking into account the mass difference of strange antiquarks, allows to get for the mass difference of bad and good diquarks, $\Delta M_{(6 F-\overline{3} F)} \sim 200 \mathrm{MeV}$, in better agreement with estimate of [58].

As it follows from the consideration of negative strangeness components of $\{27\}$ - and $\{35\}$-plets, the masses of strange quarks do not differ considerably within these multiplets, they are close to $130 \mathrm{MeV}$ and do not differ much from masses of strange valence quarks within octet and decuplet of baryons. Quite different, even paradoxical situation takes place for strange antiquark. If we take the mass of $s$-quark in antidecuplet about $(140-$ 150) $\mathrm{MeV}$, as in decuplet, then the effective mass of strange anti-quark should be small, not greater than few tens of $M e V$. For $\{27\}$-plet we obtain from (24) that strange antiquark is lighter than strange quark by $30-40 \mathrm{MeV}$, and in $\{35\}$-plet the mass of strange anti-quark is about $270-290 \mathrm{MeV}$, or about $\sim 2$ times greater than mass of strange quark within $\{27\}$-plet. Detalization of the quark models could show is it really possible, or not.

To conclude this section, we note that effective masses of strange quark and especially strange antiquark should be different for different $S U(3)$ multiplets, to make possible the link between rigid rotator version of chiral soliton and simple quark model. This issue will be considered in more details elsewhere.

\section{Partners of lowest exotic states with different spin}

The partners of lowest exotic states, i.e. the states with same flavor quantum numbers, isospin, strangeness, etc., but different spin have been discussed in the literature during latest years, after evidence has been obtained for exotic states like $\Theta^{+}$and $\Phi / \Xi_{3 / 2}$ $[56,67]$. Within $C S M$ the equality between spin of baryon states and so called "right" isospin $\left(I_{R}=1 / 2\right.$ for antidecuplet) follows from the fact that the lowest $B=1$ classical configuration is of hedgehog type, and as a result the isospin and space (or spin) rotations are equivalent. It is not so for the states with greater values of $B$ which have generally different spin and isospin [78]. At the same time, within the quark or correlated quark models one could expect existence of partners of states, since the spins of quarks and angular momentum of orbital motion can be summed providing states with different values of spin [56]. For example, according to [56] one should expect existence of partners of $\Theta^{+}$ with $J^{P}=3 / 2^{+}$and the mass greater than that of lowest states by several tens of $\mathrm{MeV}$ [56]. This possibility was considered as an argument against chiral soliton models since it was claimed that such states cannot be obtained within $C S M$.

However, careful consideration of multiplets of exotic baryons in the framework of chiral soliton approach allows to conclude that partners of lowest baryons exist within higher $S U(3)$ multiplets of baryons. Some examples are considered here.

The partners of baryon antidecuplet $\left(J^{P}=1 / 2^{+}\right)$with $J^{P}=3 / 2^{+}$exist within $\overline{35}$-plet, $(p, q)=(1,4)$, exoticness $m=2$. The rotational energy of these states is greater than that of antidecuplet, according to Table $\mathbf{1}$, by

$$
M_{\text {rot }}(\overline{35}, J=3 / 2)-M_{\text {rot }}(\overline{10}, J=1 / 2)=\frac{3}{2 \Theta_{K}}+\frac{3}{2 \Theta_{\pi}}
$$

which is about $750-800 \mathrm{MeV}$, i.e. considerably greater than quark model estimates [56]. Some contribution to the mass difference of such partners comes also from FSB mass terms, but the mass splitting between components of $\{\overline{35}\}$ corresponding to $\{\overline{10}\}$ 
is smaller than that of $\{\overline{10}\}$ itself, almost twice: the value of $\sin ^{2} \nu$ increases from $5 / 8$ to $3 / 4$, as shown in Table 6 . These states are septuquarks, at least.

\begin{tabular}{|l|l|l|l|l|l|}
\hline Baryon $\mid N(p, q), Y, I, J>$ & $<s_{\nu}^{2}>_{0}$ & $\mathrm{~A}$ & $\mathrm{~B}$ & $\mathrm{C}$ & \\
\hline$\Theta^{*} \mid \overline{35}, 2,0,3 / 2>$ & 0.625 & 2423 & 2535 & 2487 & \\
$N^{*} \mid \overline{35}, 1,1 / 2,3 / 2>$ & 0.667 & 2481 & 2586 & 2548 & \\
$\Sigma^{*} \mid \overline{35}, 0,1,3 / 2>$ & 0.708 & 2527 & 2628 & 2596 & \\
$\Xi^{*} \mid \overline{35},-1,3 / 2,3 / 2>$ & 0.750 & 2557 & 2658 & 2627 & \\
\hline \hline$N^{*} \mid 27,1,1 / 2,3 / 2>$ & 0.643 & 1739 & 1782 & 1783 & \\
$\Sigma^{*} \mid 27,0,1,3 / 2>$ & 0.679 & 1847 & 1871 & 1896 & \\
$\Lambda^{*} \mid 27,0,0,3 / 2>$ & 0.714 & 1829 & 1861 & 1876 & \\
$\Xi^{*} \mid 27,-1,1 / 2,3 / 2>$ & 0.768 & 1917 & 1937 & 1969 & \\
\hline$\Delta^{*} \mid 35,1,3 / 2,5 / 2>$ & 0.438 & 2054 & 2122 & 2137 & \\
$\Sigma^{*} \mid 35,0,1,5 / 2>$ & 0.542 & 2123 & 2181 & 2209 & \\
$\Xi^{*} \mid 35,-1,1 / 2,5 / 2>$ & 0.646 & 2186 & 2235 & 2275 & \\
$\Omega^{*} \mid 35,-2,0,5 / 2>$ & 0.750 & 2244 & 2286 & 2336 & \\
\hline
\end{tabular}

Table 6. Values of masses of the partners of the antidecuplet with $J=3 / 2$ within $\{\overline{35}\}$-plet; partners of the lowest octet within the $\{27\}$-plet, $J=3 / 2$; and partners of the lowest decuplet within the $\{35\}$-plet, $J=5 / 2$. Rigid rotator approximation has been used here according to $[45,65]$.

There are also partners of other lowest multiplets, e.g. the partners of baryons octet $J^{P}=1 / 2^{+}$with $J^{P}=3 / 2^{+}$exist, the lowest one is contained within $\{27\}$-plet with $J^{P}=3 / 2^{+}$. The difference of rotation energies equals to

$$
M_{\text {rot }}(27, J=3 / 2)-M_{\text {rot }}(8, J=1 / 2)=\frac{1}{\Theta_{K}}+\frac{3}{2 \Theta_{\pi}},
$$

so, about $580-620 \mathrm{MeV}$. More accurate numbers are presented in Table 6 . The partners of lowest $J=3 / 2$ decuplet with $J=5 / 2$ sit within $\{35\}$-plet and have the energies shown also in Table 6, last 4 lines.

Generally, within complicated $S U(3)$ multiplets, like $\{27\}$ and $\{35\}$-plets, there are also partners of iso-multiplets with same spin and different isospins. For example, within $\{27\}$-plet, for $Y=0$ there are partners with isospin $I=2, I=1$ and $I=0$, spin $J=1 / 2$ and $J=3 / 2$; for $Y=1$ there are states with $I=3 / 2$ and $1 / 2$ (see Figure). For fixed value of spin $J=I_{R}$ states with different values of isospin $I$ have same rotational energy, their mass difference is due to FSB terms, only. Such partners can be obtained within other approaches, see e.g. [60]. The spectrum of baryon states, including their partners, is rich, and interesting problem is to find correspondence with the spectrum arising from the quark models.

\section{Multibaryons with exoticness}

Numerous applications of the chiral soliton models to the properties of baryons have been widely discussed, mostly accepted and also criticized in literature. Another branch of these applications are the properties of states with baryon number greater than 1, nuclei and/or multibaryons, and this issue is much less known and accepted. The possibility to describe real nuclei as quantized chiral solitons appeared after discovery of classical chiral field configurations bound relative to the transition to states with smaller baryon numbers (history of this discovery and references can be found, e.g. in [92, 78, 93]). One 
of recent results is successful description of the mass splittings of nuclear isotopes with different values of isospin, or so called "symmetry energy" of nuclei [95]. Some variation of the only parameter of the model, Skyrme constant, allowed to provide good description of data for atomic numbers up to $\sim 30$ and to predict binding energies (b.e.) of some neutron rich nuclides [95], in general agreement with other, more traditional, approaches. The binding energies of light hypernuclei also can be calculated in general agreement with data [96]. Therefore, one can conclude that the chiral soliton approach provides results which are, at least, in qualitative agreement with existing nuclear physics data.

To obtain baryon states with definite quantum numbers, the quantization of classical configurations should be performed. There are several quantization prescriptions described in the literature to get the states with flavor quantum numbers, strangeness, charm or beauty. Besides "rigid rotator" quantization scheme originating from works [77, 39], described and used above, there is also bound state approach [89, 90] and its simplified and very transparent version $[90,91]$ which is convenient for estimating the energies of states with lowest flavor or anti-flavor quantum numbers. This scheme has been used recently to find the spectrum of lowest states with positive strangeness (beauty) or negative charm [97].

Within this quantization scheme the energy of state consists of two contributions. One, most important, is the flavor excitation energy which is of the order of $N_{c}^{0} \sim 1$. Second component is the correction of the order $\sim 1 / N_{c}$ depending on the isospin of the state (hyperfine splitting correction). In the leading order in $N_{c}$ the hamiltonian of the system can be written as

$$
H=M_{c l}+4 \Theta_{F, B} \Pi^{\dagger} \Pi+\left(\frac{3}{2} \Gamma_{S B}+\frac{N_{c}^{2} B^{2}}{16 \Theta_{F, B}}\right) D^{\dagger} D
$$

where $\Gamma_{S B}$ is given above by Eq. (15), the moment of inertia $\Theta_{F, B}=\Theta_{K}$ for $B=1$, 2-component amplitude $D$ is deviation of starting $S U(2)$ soliton into "flavor direction" which is believed to be small. Indeed, it can be easily obtained that

$$
|D| \sim\left[24 \Theta_{F, B} \Gamma_{S B}+N_{c}^{2} B^{2}\right]^{-1 / 4},
$$

i.e. it decreases with increasing $F S B$ mass and/or number of colors $N_{c}$. $\Pi$ is momentum canonically conjugate to variable $D$. The relation takes place, $D^{\dagger} D \simeq\left(1-D_{88}\right) / 3=s_{\nu}^{2} / 2$ which is fulfilled with good accuracy when $\nu$ is small.

This hamiltonian can be diagonalized and written in terms of flavor or antiflavor numbers [90, 91],

$$
H=M_{c l}+a^{\dagger} a \omega_{F, B}+b^{\dagger} b \bar{\omega}_{F, B}
$$

$a^{\dagger}, b^{\dagger}$ being flavor and antiflavor creation operators, and flavor (antiflavor) excitation energies

$$
\begin{gathered}
\omega_{F, B}=\frac{N_{c} B}{8 \Theta_{F, B}}\left(\mu_{F, B}-1\right), \quad \bar{\omega}_{F, B}=\frac{N_{c} B}{8 \Theta_{F, B}}\left(\mu_{F, B}+1\right), \\
\mu_{F, B}=\left[1+\frac{24 \Theta_{F, B} \Gamma_{S B}}{\left(N_{c} B\right)^{2}}\right]^{1 / 2} .
\end{gathered}
$$

At large $N_{c}$ the quantities $\mu_{F, B}, \omega_{F, B}$ and $\bar{\omega}_{F, B}$ scale like $N_{c}^{0} \sim 1$. When $F S B$ is small, the expansion of $\mu_{F, B}$ can be made, and the flavor excitation energy

$$
\omega_{F, B} \simeq \frac{3 \Theta_{F, B} \Gamma_{S B}}{2 N_{c} B}
$$

quadratically depends on the $F S B$ mass, because $\Gamma_{S B} \sim m_{K}^{2}$. Further details and formulas can be found in [91, 97]. 


\begin{tabular}{|l|l|l|l|l|l|l|}
\hline $\mathrm{B}$ & $\bar{\omega}_{s}$ & $\bar{\omega}_{c}$ & $\bar{\omega}_{b}$ & $\Delta \epsilon_{s}$ & $\Delta \epsilon_{c}$ & $\Delta \epsilon_{b}$ \\
\hline 1 & 591 & 1750 & 4940 & - & - & - \\
2 & 571 & 1720 & 4900 & 45 & 60 & 90 \\
3 & 564 & 1710 & 4890 & 65 & 40 & 50 \\
4 & 567 & 1710 & 4870 & 20 & 15 & 50 \\
6 & 555 & 1710 & 4880 & 55 & 30 & 40 \\
8 & 553 & 1710 & 4890 & 70 & 30 & 40 \\
12 & 547 & 1720 & 4910 & 85 & 30 & 30 \\
16 & 541 & 1720 & 4930 & 95 & 30 & 10 \\
20 & 538 & 1730 & 4940 & 100 & 20 & -10 \\
24 & 536 & 1730 & 4960 & 105 & 20 & -20 \\
\hline
\end{tabular}

Table 7. The anti-flavor excitation energies (in $M e V$ ) and binding energies changes of anti-flavored hypernuclei in comparison with ground states of ordinary nuclei (also in $\mathrm{MeV}$ ) for baryon numbers up to 24 .

The energies of anti-flavors excitation and binding energies changes of flavored multibaryons in comparison with ordinary nuclei presented in Table 7, are taken from [97]. For anti-charm and anti-beauty the antiflavor excitation energies are considerably smaller than masses of $D$ and $B$-mesons, correspondingly. It means that anti-charmed (antibeautiful) pentaquarks - their ground states - are bound relative to strong decays, since $1 / N_{c}$ corrections are small for large mass of flavor (the masses of ground state pentaquarks are $\sim 2700 \mathrm{MeV}$ for anti-charm and $5880 \mathrm{MeV}$ for $\Theta_{b}$ [97]). The property of binding of anti-charmed (-beautiful) pentaquarks is known really long ago [98, 99]. The anti-charmed pentaquark observed recently with the mass $3099 \mathrm{MeV}$ [24] can be some excitation of the ground state we discuss here, see, however [100] where the mass of the $\Theta_{c}$ was predicted to be $2985 \pm 50 \mathrm{MeV}$ within correlated quark model.

Some decrease of b.e. for anti-charm and anti-beauty, presented in Table 7, can be an artifact of approximations used to calculate them (rational map approximation [92]). For baryon numbers $B \geq 10$ the rescaled (or nuclear) variant of the model can be used, which leads to increase of b.e. in comparison with nucleon variant of the model, by several tens of $M e V$ [97]. It should be kept in mind that the mass of $\Theta^{+}$hyperon within this particular variant of the model equals to $1588 \mathrm{MeV}$. The accuracy of calculation is not better than $\sim 30-50 \mathrm{MeV}$, but deeply bound $\Theta$-hypernuclei should be expected for atomic numbers greater than $\sim 20$. Similar results have been obtained also within more traditional potential or mean-field approaches [101, 102, 103], discussion of this issue and references can be found in [104].

The increase of energy of exotic states in comparison with nonexotic ones was obtained for arbitrary $N_{c}$ within rigid rotator model as well (Appendix of [97]). It was found

$$
\Delta E_{\text {rot }}=\frac{N_{c} B+3}{4 \Theta_{F, B}}
$$

for odd $B$-numbers, and for $B=1$ this coincides with above expression, second of (10). For even $B$-numbers

$$
\Delta E_{r o t}=\frac{N_{c} B+2}{4 \Theta_{F, B}} .
$$

In derivation of these expressions it was assumed that ground states of nuclei and lowest states of flavored multibaryons belong to $S U(3)$ multiplets $(p, q)$ with the lowest possible values of $p$ [97], i.e. they have lowest allowed value of isospin - in general agreement with data. What is remarkable, the leading in $N_{c}$ contribution is the same as in rigid oscillator 
model [91] used to make calculations in [97], where the difference of anti-flavor and flavor excitation energies

$$
\bar{\omega}_{F, B}-\omega_{F, B}=\frac{N_{c} B}{4 \Theta_{F, B}} .
$$

Evidently, convergence of both quantization methods improves not only with increasing $N_{c}$, but also with increasing baryon number.

It should be noted that two different methods of quantization used in present paper, the rigid (or soft) rotator used in previous sections, and rigid oscillator method, a variant of the bound state approach [90,91], are not identical and lead to different results for $N_{c}=3$. According to (32), the mass splitting of decuplet is $\Delta_{M}(\{10\}, R O)=3 \Gamma_{S B} / 2$, whereas for rigid rotator it is 8 times smaller, according to previous results, see (14), Table 2 and also Appendix below. For the octet of baryons the RO result for total splitting is 4 times greater than the $\mathrm{RR}$ result. The $\mathrm{RO}$ method works well for exotic baryon and multibaryon states, but meets difficulties in describing the nonexotic components of $S U(3)$ multiplets which contain exotic states.

Another issue of interest could be the properties of classical chiral field configurations at large baryon numbers, as obtained within the Skyrme model. Analytical evaluations performed in [94] have shown that these properties are quite universal: at large B-numbers multibaryons described within the Skyrme model are spherical bubbles with the mass and B-number concentrated in their shell. The thickness of this shell is approximately constant, about $t \sim 3.6 /\left(F_{\pi} e\right)$, same is the average energy density in the shell, $\rho_{\text {shell }} \sim$ $F_{\pi}^{4} e^{2}$, if the mass term in the lagrangian is small enough. Both $t$ and $\rho$ do not depend on baryon number. So, the bags of matter appear in this model, the properties of "material" which these bags are made of, follow from effective chiral lagrangian [94] (in difference from traditional bag models where these properties are postulated, or introduced from phenomenological grounds). Although multibaryon configuations obtained in this way differ from ordinary nuclei, by the form of their density distribution first of all, further modifications of the model are possible, including modifications of the mass term [76, 94], but I will not go into further details here.

\section{Conclusions and prospects}

The contradictive situation with observation of pentaquark states will be resolved, probably, within next few years. Even if not all reported pentaquark states are confirmed, one could state that interesting branch of baryon spectroscopy appeared which will enlarge our knowledge about hadron structure. If none of announced observed pentaquarks is confirmed, there remains still certain theoretical interest in understanding the structure of pentaquarks and correspondence between chiral soliton and quark model descriptions. Such states can appear as broader resonances at higher energies, as it was discussed previously [31, 32, 33, 34]. Present discussion, certainly, puts more questions than gives answers. The following problems and questions can be pointed out, many of them have been, of course, noted in previous discussions $[19,18]$ :

* High statistics confirmation of existence of narrow pentaquarks seems to be necessary, especially for the resonances $\Phi / \Xi_{3 / 2}$ and $\Theta_{c}$, see [29]. Some information about experiments performed or to be performed is contained in $[29,17,19,30]$.

* Width determination is of great importance, $\Gamma \sim 1 \mathrm{MeV}$ is not excluded and suggested by analyses of scattering data, but would be difficult to explain it by theory: a special reason is necessary then.

* Several missing components of multiplets remain to be found, for example, of considerable interest are:

in $\{\overline{10}\}$-plet: $\Phi / \Xi_{3 / 2}^{+} \rightarrow \Xi^{0} \pi^{+}, \quad \Sigma^{+} \bar{K}^{0}$; 
in $\{27\}$-plet: $\Theta_{1}^{*} \rightarrow N K ; \Xi_{3 / 2}^{*} \rightarrow \Xi \pi ; \Sigma_{2} \rightarrow \Sigma \pi ; ; \Omega_{1} \rightarrow \Omega \pi, \Xi \bar{K}$;

in $\{35\}$-plet: $\Omega_{1}^{*} \rightarrow \Omega \pi, \Xi \bar{K}, \Xi^{*} \bar{K} ; \Delta_{5 / 2} \rightarrow N \pi \pi ; \Gamma_{S=-4} \rightarrow \Omega \bar{K}$, etc.

In the latter case the complication is due to the fact that most of interesting components of $\{35\}$-plet are not available in octet-octet meson-baryon interaction.

* Studies of cryptoexotics $\left(N^{*}, \Delta^{*}, \Xi^{*} \ldots\right)$ are of interest as well, to complete the picture of pentaquarks, more detailed discussion can be found in $[18,82]$.

* Spin and parity are crucial for cheking the validity of chiral soliton models predictions. Negative parity of these states would provide big difficulties for their interpretation as quantized topological solitons, although in any model it seems unrealistic to get a narrow resonance, with $\Gamma \leq 10 \mathrm{MeV}$, decaying into S-wave state of meson and baryon with energy release about $100 \mathrm{MeV}$.

* As a result, better understanding of the structure of baryons and their wave functions will be reached. The understanding of the possible important role of correlated diquarks and triquarks in the baryons wave functions could be the first example [55,61]. The link of the soliton approach and quark models leads to the conclusion that the effective masses of strange quark and antiquark within baryon states should be considerably different and depend on the particular $S U(3)$ multiplet, and this can be another example. The difference of masses of strange antiquarks within 27-plet and 35-plet is so large, that it looks as paradox.

* As it was noted in literature [66, 68], predictions of chiral soliton models are not completely selfconsistent from the point of view of the $1 / N_{c}$ expansion. In addition to problems considered in [66, 68], we note, e.g., that the mass splitting between octet and antidecuplet of baryons is of the order of $N_{c}^{0} \sim 1$, whereas the total mass splitting within octet or antidecuplet is of the order of $N_{c}$, as the classical soliton mass itself. There is also some inconsistency between rigid rotator and bound state quantization models, in particular the mass splittings within $S U(3)$ multiplets given by these models, differ considerably. These mass splittings coincide at large $N_{c}$, in the leading in $1 / N_{c}$ approximation, but for $N_{c}=3$ the bound state approach in its present form (the rigid oscillator model, in particular [91]) gives much greater splittings when FSB mass is not large (see Appendix). In view of these difficulties, the results obtained in the large $N_{c}$ limit, including e.g. some objections against chiral soliton model results [66, 68], should be interpreted with great care and may not be valid for the real $N_{c}=3$ world. Predictions of chiral soliton approach should be considered as a reasonable extrapolation, when one of states of interest is fitted. Results of this extrapolation are impressive sometime.

* Other predictions of CSM are of interest besides those discussed in present paper, e.g. supernarrow radiatively decaying dibaryon (JINR and INR experiments [105, 106], see, however, [107] where negative result was obtained for low values of dibaryon masses).

* Chiral soliton models are good example of the field theoretical models which allow to obtain the results of practical interest. They provide a possibility to describe not only baryons and baryonic resonances, but also systems with large baryon numbers as "bags" of certain type, the properties of these bags are deduced from initial lagrangian [94].

Some important and interesting issues have not been considered here in view of restricted size of the paper: exotic baryons production mechanisms and their properties; determination of spin-parity and electromagnetic properties of these baryons, etc. Discussion and references can be found in [64, 18].

Acknowledgments. Computer programs for configuration mixing arranged by Bernd Schwesinger and Herbert Weigel (soft rotator approximation) and by Hans Walliser (rigid rotator) have been used in present paper. I'm greatly indebted to Hans Walliser also for his help in numerical calculations, useful remarks and criticism, to Tom Cohen and Igor Klebanov for helpful discussion of large $N_{c}$ subtleties, to Igor Strakovsky for reading the manuscript and useful comments on experimental data available now, and to Andrei Shunderuk for checking of some formulas and numerical results. E-mail conversa- 
tions with Ya.I.Azimov, D.I.Diakonov, G.Holzwarth, R.L.Jaffe, S.Kabana, J.Trampetic, H.Weigel are acknowledged, as well as discussions with A.A.Andrianov, V.A.Andrianov, L.N.Bogdanova, K.G.Boreskov, B.L.Ioffe, B.Z.Kopeliovich, L.N.Lipatov, V.M.Lobashev, V.A.Matveev, Yu.V.Novozhilov, L.B. Okun', V.A.Rubakov, Yu.A.Simonov, K.A. TerMartirosyan.

\section{Appendix. Some properties of the large $N_{c}$ baryons.}

As it was discussed above, at large arbitrary (odd) number of colors $N_{c}$ baryon consists of $N_{c}$ quarks in color singlet state, and there are totally $\left(N_{c}+1\right) / 2$ nonexotic $S U(3)$ multiplets of baryons, from $[p, q]=\left[1,\left(N_{c}-1\right) / 2\right]$, to $[p, q]=\left[N_{c}, 0\right]$. The hypercharge for arbitrary $N_{c}$ is $Y=N_{c} B / 3+S([39]$, see also $[68,66])$. One possibility for the choice of charges of quarks is as usual, $Q_{u}=2 / 3, Q_{d}=Q_{s}=-1 / 3$, see [66] e.g. In this case the electric charge defined by relation $Q=I_{3}+Y / 2$ is integer only if $N_{c}$ is multiple of 3. Another possibility for electric charges was discussed in [87]. where the supercharged quarks and $S U(3)$ multiplets were considered. In this case $Q=I_{3}+Y / 2+B\left(3-N_{c}\right) / 6$, the charges of quarks follow from this expression at $B=1 / N_{c}$ :

$$
Q_{u}=\frac{1}{2}+\frac{1}{2 N_{c}}, \quad Q_{d}=Q_{s}=-\frac{1}{2}+\frac{1}{2 N_{c}}
$$

and average charge of each baryonic $S U(3)$ multiplet, or supercharge, equals to $\bar{Q}=$ $\left(3-N_{c}\right) B / 6$.

Below strangeness contents of baryons at large number of colors are presented, which define the mass splittings of baryon multiplets within rigid rotator approximation. For the multiplet $[p, q]=\left[1,\left(N_{c}-1\right) / 2\right]$ which is analogue of the $N_{c}=3$ octet we obtained:

$$
\begin{aligned}
& <s_{\nu}^{2}>{ }{ }_{N "}=\frac{4\left(N_{c}+4\right)}{\left(N_{c}+3\right)\left(N_{c}+7\right)}, \quad<s_{\nu}^{2}>{ }{ }^{\prime \prime}=\frac{6}{\left(N_{c}+7\right)}, \\
& <s_{\nu}^{2}>{ }{ }_{\Sigma}, \quad=\frac{2\left(3 N_{c}+13\right)}{\left(N_{c}+3\right)\left(N_{c}+7\right)}, \quad<s_{\nu}^{2}>{ } \Xi^{\prime \prime}=\frac{8}{\left(N_{c}+7\right)} \text {. }
\end{aligned}
$$

Evidently, the Gell-Mann-Okubo relation $M_{\Sigma}+3 M_{\Lambda}=2\left(M_{N}+M_{\Xi}\right)$ is fulfilled for these values of $\left\langle s_{\nu}^{2}\right\rangle$. The increase of $\left\langle s_{\nu}^{2}>\right.$ per unit of strangeness within "octet" of baryons equals, in average, at large $N_{c}$

$$
\Delta\left(<s_{\nu}^{2}>, \delta|S|=1, " 8 "\right) \simeq \frac{2}{N_{c}}\left(1-\frac{8}{N_{c}}\right) .
$$

For the components of "decuplet" $[p, q]=\left[3,\left(N_{c}-3\right) / 2\right]$ we obtain

$$
\begin{aligned}
& <s_{\nu}^{2}>{ }^{\prime} \Delta^{\prime \prime}=\frac{4\left(N_{c}+4\right)}{\left(N_{c}+1\right)\left(N_{c}+9\right)}, \quad<s_{\nu}^{2}>{ }{ }_{\Sigma *} "=\frac{2\left(3 N_{c}+7\right)}{\left(N_{c}+1\right)\left(N_{c}+9\right)}, \\
& <s_{\nu}^{2}>" \Xi * "=\frac{4\left(2 N_{c}+3\right)}{\left(N_{c}+1\right)\left(N_{c}+9\right)}, \quad<s_{\nu}^{2}>" \Omega^{\prime \prime}=\frac{10}{\left(N_{c}+9\right)}, .
\end{aligned}
$$

Equidistant behaviour of "decuplet" components can be noted, with a step

$$
\begin{aligned}
\Delta\left(<s_{\nu}^{2}>, \delta|S|=1, " 10 "\right) & =2\left(N_{c}-1\right) /\left[\left(N_{c}+1\right)\left(N_{c}+9\right)\right] \\
& \simeq \frac{2}{N_{c}}\left(1-\frac{11}{N_{c}}+\frac{101}{N_{c}^{2}}\right) .
\end{aligned}
$$


At large $N_{c}$ average splittings within "octet" and "decuplet" coincide and equal to $2 / N_{c}$, but preasymptotic corrections $\sim 1 / N_{c}^{2}$ are different, making splitting within "decuplet" smaller than within "octet", in qualitative agreement with observations for octet and decuplet in real world.

For the $\Theta^{+} \in "\{\overline{10}\} "$ it is easy to obtain

$$
<s_{\nu}^{2}>{ }^{\prime} \Theta^{\prime}=\frac{6}{\left(N_{c}+9\right)},
$$

and for the $\Theta_{1} \in "\{27\} "$

$$
<s_{\nu}^{2}>{ }^{{ }{ }{ }^{\prime}}=\frac{2(3 N+23)}{\left(N_{c}+5\right)\left(N_{c}+11\right)},
$$

which is slifgtly greater than for $\Theta$.

At large $N_{c}$ it is a matter of simple algebra to establish that there is equidistant behaviour of strangeness contents (recall that $\left\langle s_{\nu}^{2}\right\rangle_{B}=2 S C_{B}$ ) for the components of non-exotic multiplets, with not large (fixed) values of strangeness $S$ :

$$
<s_{\nu}^{2}>_{\left(p \sim 1, q \sim N_{c} / 2,|S| \sim 1\right)} \simeq 2 \frac{2+|S|}{N_{c}} .
$$

As a result, in the limit $N_{c} \rightarrow \infty$ the mass splittings between adjacent components of "octet", "decuplet" and other nearest multiplets coincide with those obtained within rigid oscillator approximation [91]. However, $1 / N_{c}^{2}$ corrections to these asymptotic values of mass splittings are large, the expansion parameter is about $\sim 11 / N_{c}$ or $\sim 8 / N_{c}$. These corrections lead to the decrease of the mass splittings between adjacent components of these multiplets, and this effect becomes of the order 1 when $|S| \sim N_{c}$. As a result, the total mass splittings of the whole multiplets are smaller than within RO approximation by numerical factors. For the real world, $N_{c}=3$, the mass splitting of decuplet is 8 times smaller for rigid rotor approximation than for rigid oscillator (when FSB mass is small and hyperfine splitting correction of the order $\sim 1 / N_{c}$ is not included), and 4 times smaller for the octet. Hyperfine splitting correction as found in [90, 91] decreases the mass splitting within $\mathrm{RO}$ model, but not sufficient. It is possible to modify the next to leading in $1 / N_{c}$ contributions to the mass splittings by means of appropriate resolution of the operator ordering ambiguity within $\mathrm{RO}$ and to remove the difference from $\mathrm{RR}$ model [108]. However, it is not clear how to make extrapolation to realistic value $N_{c}=3$, and what is the influence of such modification on results and conclusions of paper [68]. This illustrates well that although both methods, $\mathrm{RR}$ and RO, converge at large $N_{c}$, small $m_{K}$ and fixed not large values of strangeness, in real world there is considerable difference between both approaches.Recently the paper [109] echoed the difficulties of extrapolation of results obtained in large $N_{c}$ world to the realistic $N_{c}=3$ world, at least for some physical observables

When $m_{K}$ is large (as for charm or beauty quantum numbers) the flavor excitation energies for RO method depend linearly on $m_{D}$, which looks much more realistic than for $\mathrm{RR}$ method, and the bound state approach $[89,91]$ is more preferable.

NOTES ADDED in PROOF. The higher statistics study of the positively charged kaon interactions in the Xe bubble chamber [110] reinforced the evidence of the DIANA Collaboration for the production of the $\Theta^{+}$hyperon, with the confidence level from 4.3 to 7.3 standard deviations, depending on the estimation method. At the same time, the CLAS Collaboration in recent high statistics experiment [111] disavowed their previous result on the observation of $\Theta^{+}$in the photoproduction reaction on deuterons. 
Detailed calculations of the strangeness contents of all components of exotic multiplets of baryons (pentaquarks) have been performed recently at an arbitrary number of colors $N_{c}$ [112] within the rigid rotator model. The leading terms in the $1 / N_{c}$ expansion for the positive strangeness states coincide with those in the rigid oscillator model, but the next-to-leading order terms differ essentially from those obtained within the RO model in its commonly accepted version, as for the "octet" and "decuplet" of baryons discussed in [108] and in present paper.

\section{References}

1. PDG Collab., Phys. Lett. B592 (2004); J. Phys. G 33, 1 (2006)

2. T. Nakano et al (LEPS Collab.), Phys. Rev. Lett. 91, 012002 (2003); hep-ex/0301020

3. V.V. Barmin et al (DIANA Collab.) Yad. Fiz. 66, 1763 (2003); hep-ex/0304040

4. S. Stepanyan et al (CLAS Collab.) Phys. Rev. Lett. 91, 252001 (2003)

5. J. Barth et al (SAPHIR Collab.) hep-ex/0307083 ; Phys. Lett. B572, 127 (2003)

6. A.E. Asratyan, A.G. Dolgolenko and M.A. Kubantsev, Yad.Fiz. 67, 704 (2004)

7. V. Kubarovsky et al (CLAS Collab.) Phys. Rev. Lett. 92, 032001 (2004)

8. S. Chekanov et al (ZEUS Collab.) Phys. Lett. B591, 7 (2004)

9. A. Airapetian et al (HERMES Collab.) Phys. Lett. B585, 213 (2004)

10. A. Aleev et al (SVD Collab.) hep-ex/0401024, Phys. Atom. Nucl. 68, 974 (2005)

11. M. Abdel-Bary et al (COSY-TOF Collab.) hep-ex/0403011; Phys. Lett. B595, 127 (2004)

12. Yu.A. Troyan et al (JINR $H_{2}$ bubble chamber Collab.), hep-ex/0404003

13. M. Battaglieri, R. De Vita, talk at the meeting NSTAR-2004

14. R. Arndt, I. Strakovsky and R. Workman, Phys. Rev. C68, 042201 (2003); R. Workman, R. Arndt, I. Strakovsky, D. Manley and J. Tulpan, Phys. Rev. C70, 028201 (2004)

15. R.N. Cahn and G.H. Trilling, Phys. Rev. D69, 011501 (2004)

16. J. Pochodzalla, Pentaquarks: facts and mysteries, or Sisyphus at work. hep-ex/0406077

17. K. Hicks, Workshop Summary: Experiment, hep-ex/0501018

18. Ya.I. Azimov, R.A. Arndt, I.I. Strakovsky, R.L. Workman and K. Goeke, Eur. Phys. J. A26, 79 (2005)

19. K.H. Hicks, Experimental search for pentaquarks, Prog. Part. Nucl. Phys. 55, 647 (2005)

20. M. Battaglieri, R. De Vita, V. Kubarovsky, D. Weygand and the CLAS Collab., Talk at the APS Meeting, April 16 (2005)

21. C. Alt et al (NA49 Collab.) Phys. Rev. Lett. 92, 042003 (2004); hep-ex/0310014

22. E.S. Ageev et al (COMPASS Collab.) hep-ex/0503033, Eur. Phys. J. C41, 469 (2005)

23. J. Spengler (for HERA-B Collab.) hep-ex/0504038, Acta Phys. Polon. B36, 2223 (2005)

24. A. Aktas et al (H1 Collab.) Phys. Lett. B588, 17 (2004); hep-ex/0403017 ; C. Risler (for H1 Collab.), hep-ex/0506077

25. U. Karshon (for ZEUS Collab.) hep-ex/0407004; hep-ex/0410029; S. Chekanov et al (ZEUS Collab.) hep-ex/0409033; Eur. Phys. J. C38, 29 (2004)

26. M. Ripani et al (CLAS Collab.), Phys. Rev. Lett. 91, 022002 (2003); hep-ex/0304034

27. S. Kabana (for STAR Collab.) hep-ex/0406032

28. P. Aslanyan, V. Emelyanenko and G. Rikhvitskaya, hep-ex/0504026

29. V. Kubarovsky, P. Stoler (for CLAS Collab.) hep-ex/0409025; P. Rossi, Nucl. Phys. A752, 111 (2005)

30. S. Kabana, AIP Conf. Proc. 739, 181 (2005); ibid. 756, 195 (2005)

31. R.L. Jaffe, SLAC-PUB-1774 ; Phys. Rev. D15, 267, 281 (1977)

32. H. Hogaasen and P. Sorba, Nucl. Phys. B145, 119 (1978)

33. D. Strottman, Phys.Rev. D20, 748 (1979)

34. C. Roiesnel, Phys. Rev. D20, 1646 (1979)

35. A. Manohar, Nucl. Phys. B248, 19 (1984); M. Chemtob, Nucl.Phys. B256, 600 (1985) 
36. L.S. Biedenharn and Y. Dothan, From SU(3) to Gravity (Ne'eman Festschrift), Cambridge University Press, Cambridge (1986)

37. M. Karliner and M.P. Mattis, Phys. Rev. D34, 1991 (1986)

38. M. Praszalowicz, Proc. of the Workshop Skyrmions and Anomalies Krakow, Poland, 20-24 Febr. 1987, World Scientific, Ed. M.Jezabek, M.Praszalowicz, p.112.

39. E. Guadagnini, Nucl.Phys. B236, 35 (1984)

40. V. Kopeliovich, On exotic systems of baryons in chiral soliton models. NORDITA Preprint 90/55 NP (1990); Phys. Lett. B259, 234 (1991)

41. E. Jenkins and A.V. Manohar, hep-ph/0401190, Phys. Rev. Lett. 93, 022001 (2004); hepph/0402150, Phys. Rev. D70, 034023 (2004)

42. H. Walliser, An Extension of the Standard Skyrme model, Proc. of the Workshop Baryon as Skyrme Soliton, Siegen, Germany, 28-30 Sept. 1992, World Scientific, Ed. G.Holzwarth, p.247; Nucl. Phys. A548, 649 (1992)

43. D. Diakonov, V. Petrov and M. Polyakov, Z. Phys. A359, 305 (1997)

44. H. Weigel, Eur. Phys. J. A2, 391 (1998)

45. H. Walliser and V.B. Kopeliovich, JETP 97, 433 (2003) ; hep-ph/0304058

46. M. Praszalowicz, Phys. Lett.B575, 234 (2003); hep-ph/0308114

47. D. Borisyuk, M. Faber and A. Kobushkin, hep-ph/0307370; Ukr. J. Phys. 49, 944 (2004)

48. M. Praszalowicz, Phys. Lett. B583, 96 (2004); hep-ph/0311230; Acta Phys. Polon. B35, 1625 (2004); hep-ph/0402038

49. B. Wu and B.Q. Ma, hep-ph/0312041; hep-ph/0408121

50. J. Ellis, M. Karliner and M. Praszalowicz, JHEP 0405002 (2004)

51. H. Weigel, Eur. Phys. J. A21, 133 (2004); hep-ph/0404173; hep-ph/0405285

52. G. Duplancic, H. Pasagic and J. Trampetic, Phys. Rev. D70, 077504 (2004); JHEP 0407, 027 (2004)

53. H.K. Lee and H.Y. Park, hep-ph/0406051

54. M. Karliner and H.J. Lipkin, hep-ph/0307243; Phys. Lett. B575, 249 (2003)

55. R. Jaffe and F. Wilczek, Phys. Rev. Lett. 91, 232003 (2003); hep-ph/0307341

56. F. Close, The End of the Constituent Quark model?, Summary talk at the Baryon 03 Conf., AIP Conf. Proc. 717, 919 (2004), hep-ph/0311087

57. J.J. Dudek and F.E. Close, Phys. Lett. B583, 278 (2004), hep-ph/0311258; F.E. Close and J.J. Dudek, Phys. Lett. B586, 75 (2004), hep-ph/0401192

58. F. Wilczek, Diquarks as Inspiration and as Objects, hep-ph/0409168

59. F. Close, Hadron Spectroscopy (theory): Diquarks, Tetraquarks, Pentaquarks and no quarks, Plenary talk at 32nd International Conf. on High Energy Physics, ICHEP 04, Bejing, China, August 2004, hep-ph/0411396

60. S.-L. Zhu, Phys. Rev. Lett. 91, 232002 (2003); B.L. Ioffe and A.G. Oganesian, Pis'ma v ZhETF 80, 439 (2004), hep-ph/0408152; R.D. Matheus and S. Narison, hep-ph/0412063;

61. N.I. Kochelev, H.-J. Lee and V. Vento, Phys. Lett. B594, 87 (2004); A.E. Dorokhov and N.I. Kochelev, hep-ph/0411362; H-J. Lee, N.I. Kochelev and V. Vento, Phys. Lett. B610, 50 (2005), hep-ph/0412127; hep-ph/0506250

62. F. Csikor et al, hep-lat/0309090; JHEP 0311, 070 (2003); hep-lat/0503012

63. S. Sasaki, Phys. Rev. Lett. 93, 152001 (2004); hep-lat/0410016; T.-W. Chiu and T.-H. Hsieh, hep-ph/0403020

64. B.K. Jennings, K. Maltman, Phys. Rev. D69, 094020 (2004), hep-ph/0308286 ; D. Diakonov, hepph/0406043; S.-L. Zhu, hep-ph/0406204; M. Oka, hep-ph/0406211; M. Karliner, Acta Phys. Polon. B35, 3055 (2004); Int. J. Mod. Phys. A20, 199 (2005); R.L. Jaffe, Phys. Rep. 409, 1 (2005); M. Praszalowicz and K. Goeke, hep-ph/0506041; A. Hosaka, hep-ph/0506138; I.M. Narodetsky, Yad. Fiz. 68, 780 (2005)

65. V. Kopeliovich, Physics Uspekhi, 47, 309 (2004) ; hep-ph/0310071

66. T. Cohen, Phys. Lett. B581, 175 (2004), hep-ph/0309111; hep-ph/0312191

67. T.D. Cohen and R.F. Lebed, Phys. Lett. B578, 150 (2004), hep-ph/0309150 
68. N. Itzhaki, I.R. Klebanov, P. Ouyang and L. Rastelli, Nucl. Phys. B684, 264 (2004); hep$\mathrm{ph} / 0309305$

69. T.H.R. Skyrme, Proc. Roy. Soc. A260, 127 (1961)

70. E. Witten, Nucl. Phys. B223, 433 (1983)

71. D.I. Diakonov and M. Eides, JETP Lett. 38, 433 (1983)

72. J. Balog, Phys. Lett. B149, 197 (1984)

73. A.A. Andrianov, V.A. Andrianov, Yu. V. Novozhilov and V.Yu. Novozhilov, Lett. Math. Phys. 11, 217 (1986); Phys. Lett. B186, 401 (1987); ibid. B203, 160 (1988)

74. A. Jackson et al, Phys. Lett. B154, 101 (1985)

75. L. Marleau, Phys. Lett. B235, 141 (1990); Phys. Rev. D45, 1776 (1992); L. Marleau and J-F. Rivard, Phys. Rev. D63, 036007 (2001)

76. L. Marleau, Phys. Rev. D43, 885 (1991)

77. G. Adkins, C. Nappi and E. Witten, Nucl. Phys. B228, 552 (1983)

78. V.B. Kopeliovich, Sov.J.Nucl.Phys. 47, 949 (1988); E. Braaten and L. Carson, Phys. Rev. D38, $3525(1988)$

79. D. Diakonov, V. Petrov, Phys. Rev. D69, 094011 (2004)

80. M.M. Pavan, R.A. Arndt, I.I. Strakowsky and R.L. Workman, Proc. of the 9th International Symposium on Meson-Nucleon Physics and Structure of Nucleon (MENU2001), Washington, DC, USA, July 26-31, 2001, ed. by H. Haberzettl and W. Briscoe, $\pi \mathrm{N}$ Newslett. 16, 110 (2002); hep-ph/0111066

81. V. Kuznetsov (for GRAAL Collab.), hep-ex/0409032

82. R.A. Arndt, Ya.I. Azimov, M.V. Polyakov, I.I. Strakovsky and R.L. Workman, Phys. Rev. C69, 035208 (2004), nucl-th/0312126

83. R.A. Arndt et al, Phys. Rev. C69, 035213 (2004), nucl-th/0311089

84. B. Schwesinger and H. Weigel, Phys. Lett. B267, 438 (1991)

85. B. Moussalam, Ann. of Phys. (N.Y.) 225, 264 (1993); F. Meier, H. Walliser, Phys. Rept. 289, 383 (1997)

86. V. Kopeliovich, B. Schwesinger and B. Stern, Nucl. Phys. A549, 485 (1992)

87. A. Abbas, Phys. Lett. B238, 344 (1990); Phys. Lett. B503, 81 (2001); H. Walliser, unpublished.

88. Y. Oh and H. Kim, Phys. Rev. D70, 094022, (2004), hep-ph/0405010; hep-ph/0409358

89. C.G. Callan and I.R. Klebanov, Nucl. Phys. B262, 365 (1985); N. Scoccola et al, Phys. Lett. B201, 425 (1988)

90. D. Kaplan and I.R. Klebanov, Nucl. Phys. B335, 45 (1990)

91. K.M. Westerberg and I.R. Klebanov, Phys. Rev. D50, 5834 (1994)

92. C. Houghton, N. Manton and P. Sutcliffe, Nucl. Phys. B510, 507 (1998)

93. V.B. Kopeliovich, W.J. Zakrzewski, JETP Lett, 69, 721 (1999); Eur. Phys. J C18, 369 (2000); V.B. Kopeliovich, JETP, 93, 435 (2001)

94. V.B. Kopeliovich, J. Phys. G28, 103 (2002); JETP Lett. 73, 587 (2001); V. Kopeliovich, B. Piette and W. Zakrzewski, hep-th/0503127

95. V. Kopeliovich, A. Shunderuk and G. Matushko, nucl-th/0404020, Yad. Fiz. (2005) in press.

96. V.B. Kopeliovich, JETP 96, 782 (2003); nucl-th/0209040

97. V. Kopeliovich and A. Shunderuk, JETP 100, 929 (2005), nucl-th/0409010

98. D.O. Riska and N.N. Scoccola, Phys. Lett. B299, 338 (1993)

99. Y. Oh, B.Y. Park and D.P. Min, Phys. Lett. B331, 362(1994); Phys. Rev. D50, 3350 (1994)

100. M. Karliner and H.J. Lipkin, hep-ph/0307343

101. D. Cabrera et al, Phys. Lett. B608, 231 (2005)

102. X. Zhong et al, Phys. Rev. C71, 015206 (2006)

103. M.J. Vicente Vacas et al, nucl-th/0410056

104. D.E. Lanskoy, nucl-th/0411004

105. A. Khrykin et al, Phys. Rev. C64, 034002 (2001)

106. L.V. Filkov et al, Eur. Phys. J. A12, 369 (2001)

107. A. Tamii et al, Phys. Rev. C65, 047001 (2002) 
108. I.R. Klebanov and V.B. Kopeliovich, "Large $N_{c}$ comparison of the rigid oscillator and rotator approaches to Skyrmions", June 2005, unpublished notes.

109. T.D. Cohen, P.M. Hohler and R.F. Lebed, Phys. Rev. D72, 074010 (2005)

110. V.V. Barmin et al. (DIANA Collab.), hep-ex/0603017

111. B. VcKinnon et al. (CLAS Collab.), Phys. Rev. Lett. 96, 212001 (2006)

112. V.B. Kopeliovich and A.M. Shunderuk, Phys. Rev. D73, 094018 (2006) 

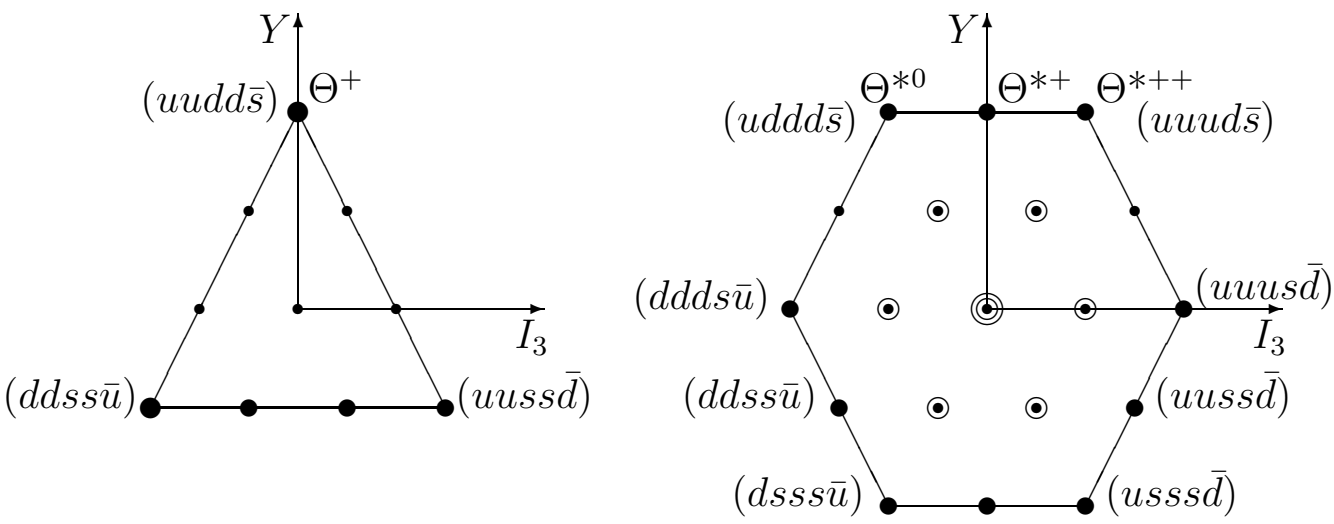

$\{\overline{10}\} J=1 / 2$

$\{27\} J=3 / 2 ; 1 / 2$

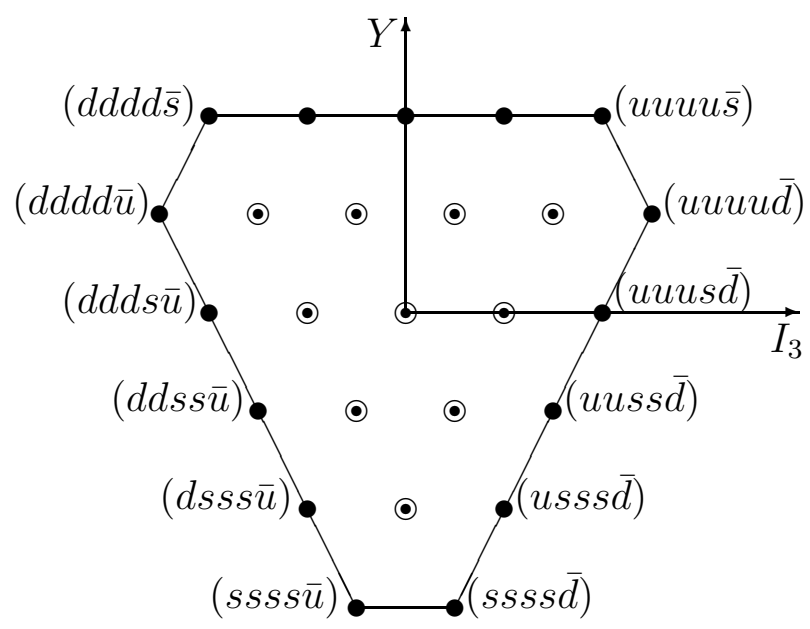

$\{35\} J=5 / 2 ; 3 / 2$

Figure 1: The $I_{3}-Y$ diagrams for the multiplets of pentaquarks, $B=1, m=1$. Large full circles show the exotic states, smaller - the cryptoexotic states which can mix with nonexotic states from octet and decuplet. Manifestly exotic components of pentaquarks satisfy the relation $I=(5+S) / 2$ for strangeness $S \leq 0$ and have unique quark contents shown in this figure. 